\title{
Coupled thermally general imperfect and mechanically coherent energetic interfaces subject to in-plane degradation
}

\author{
A. Esmaeili ${ }^{\mathrm{a}}$, P. Steinmann ${ }^{\mathrm{a}}$, A. Javili ${ }^{\mathrm{b}, *}$ \\ ${ }^{a}$ Chair of Applied Mechanics, University of Erlangen-Nuremberg, Egerlandstrasse 5, 91058 Erlangen, Germany \\ ${ }^{b}$ Department of Mechanical Engineering, Bilkent University, 06800 Ankara, Turkey
}

\begin{abstract}
To date, the effects of interface in-plane damage on the thermo-mechanical response of a thermally general imperfect (GI) and mechanically coherent energetic interface are not taken into account. A thermally GI interface allows for a discontinuity in temperature as well as in the normal heat flux across the interface. A mechanically coherent energetic interface permits a discontinuity in the normal traction but not in the displacement field across the interface. The temperature of a thermally GI interface is a degree of freedom and is computed using a material parameter known as the sensitivity. The current work is the continuation of the model developed in [21] where a degrading highly-conductive (HC) and mechanically coherent energetic interface is considered. An HC interface only allows for the jump in normal heat flux and not the jump in temperature across the interface. In this contribution, a thermodynamically consistent theory for thermally general imperfect and mechanically coherent energetic interfaces subject to in-plane degradation is developed. A computational framework to model this class of interfaces using the finite element method is established. In particular, the influence of the interface in-plane degradation on the sensitivity is captured. To this end the equations governing a fully non-linear transient problem are given. They are solved using the finite element method. The results are illustrated through a series of three-dimensional numerical examples for various interfacial parameters. In particular, a comparison is made between the results of the intact and the degraded thermally GI interface formulation.
\end{abstract}

Keywords: Thermo-mechanically energetic interfaces, Interface elasticity, General imperfect (GI) interfaces, Non-local damage, Nanomaterials, Finite element method.

\section{Introduction}

Interfaces possess different thermo-mechanical properties from those of the bulk which becomes dominating as the length scale reduces. Note that the smaller the scale, the larger the interface area to bulk volume ratio [8, 16, 19].

\footnotetext{
${ }^{*}$ Corresponding author

Email addresses: ali.esmaeili@fau.de (A. Esmaeili), paul.steinmann@ltm.uni-erlangen.de (P. Steinmann), ajavili@bilkent.edu.tr (A. Javili) 
This dominating influence motivates one to devise a more realistic model to better capture the physics of interface materials. In addition, the followings are a few additional motivations to develop a more general interface model:

- increasing applications of thermal interfaces [38],

- unusual thermal behavior of surface and interfaces at the nano-scale [6, 7, 10, 37],

- the study of interface mechanical characteristics by the vast majority of the literature is mainly based on cohesive zone models.

Therefore, in this contribution, we follow the work of [28,33] where the interface theory was extended to mechanically coherent energetic and thermally general imperfect (GI) interfaces. A thermally general imperfect (GI) interface permits discontinuities in both temperature and normal heat flux. The extreme cases of thermally GI interfaces are highly conductive (HC) and lowly conductive (LC) interfaces, where the former allows a discontinuity in the normal heat flux but not in the temperature across the interface, and the latter permits a discontinuity in the temperature but not in the normal heat flux across the interface. Among the various thermal interfaces introduced above, a HC interface is termed thermally coherent due to the vanishing temperature jump. We point out that the thermally GI interface presented here may be specialized to all the other types of thermal interfaces. For further details on the different types of thermal imperfections see [20, 21, 28, 33] and references therein.

A mechanically coherent energetic interface is based on the interface elasticity theory proposed by [25, 36]. Note that the coherence of the interface refers to the continuity in the displacement field across the interface. This manuscript is limited to mechanically coherent energetic interfaces. For further details see for instance, [1, 4, 5, 11, $12,15,-19,22,-24,26,27,31,34,35,40,42,46]$ and references therein. A restriction of the interface elasticity theory is that it only captures elastic interface behavior.

The nucleation of micro-voids and strong discontinuities such as cracks can act as shields or amplify stress intensity in other regions of an interface. Consequently, this can influence the temperature distribution and thus the thermo-mechanical response of a body. Noting that the interface elasticity theory can only capture elastic behavior of energetic interfaces, the development of a more general interface model, in which interface inelasticity is taken into account, seems necessary.

Very recently in [21] we have considered thermally highly-conductive (HC) interfaces in a thermomechanical body, whereby due to the highly conductive property the (otherwise mechanically coherent) interfaces allow for jumps in the normal heat flux. Moreover they are equipped with interface stresses that are coupled to in-plane damage. In this contribution, we formulate a follow up version of [21] that generalizes the thermal part of the above interfaces to the thermally general imperfect case, whereas the mechanical part is as before. Thereby this formulation embraces the 
two limiting cases of the previous HC interfaces (jump in normal heat flux) and the lowly-conductive (LC) Kapitza interfaces [see 20] that allow for a jump in the temperature. Thus arbitrary combinations of HC and LC (jump in normal heat flux and jump in the temperature) are analyzed numerically.

(a)



thermally GI interface (b)

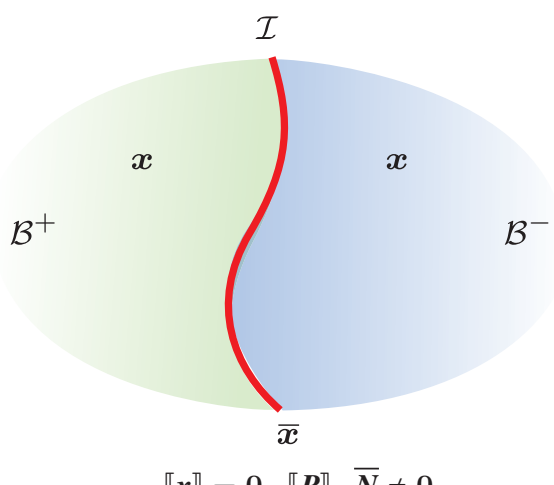

$\llbracket x \rrbracket=0, \llbracket P \rrbracket \cdot \bar{N} \neq 0$

mechanically coherent energetic interface

Figure 1: (a) Thermally general imperfect interface and (b) mechanically coherent energetic interface. The interface in this work is mechanically coherent, thus no jump in deformation is allowed across the interface, $\llbracket \varphi \rrbracket=\mathbf{0}, \llbracket \boldsymbol{x} \rrbracket=\mathbf{0}$, and energetic, thus the jump of the normal traction across the interface does not vanish, i.e. $\llbracket \boldsymbol{P} \rrbracket \cdot \overline{\boldsymbol{N}} \neq \mathbf{0}$. A thermally GI interface allows for the jump in temperature $\llbracket \Theta \rrbracket \neq \mathbf{0}$, and in normal heat flux $\llbracket Q \rrbracket \cdot \bar{N} \neq 0$ across the interface. The bulk and interface are denoted here by $\mathcal{B}$ and $\mathcal{I}$. The normal to the interface is denoted by $\overline{\boldsymbol{N}}$, see Fig. 2 The in-plane degradation of the interface causes the degradation of mechanical and thermal properties of the interface through a tangential damage variable $\bar{D}_{\|}$.

To take into account the in-plane damage, a non-local continuum damage approach is utilized. For further details on this approach with application to bulk materials see for instance [9, 13, 14, 32, 39, 45], among others. There are a few reasons to use a non-local damage model: first, mesh-objective finite element simulation of strain softening materials; second, determination of growth of micro-cracks by the energy release from the volume encompassing the micro-crack [3]; third, the influence of the presence of a micro-crack on the stress level of other neighboring micro-cracks; and finally capturing size effects [2]. The non-locality in this work is of integral-type which then requires the use of an interactive (cut-off) radius, capturing size effects. The interactive radius is a function of the molecular structure of the intact material and distribution and growth of the micro-cracks in the damaging material. It is not yet well-established how to determine the interactive radius from experiments. See [2] for further details. The degradation of the interface material here is measured using a tangential (in-plane) damage variable denoted by $\bar{D}_{\|}$. Consequently, as the damage variable evolves all the mechanical and in-plane thermal properties of the interface are reduced. However, the out-of-plane thermal properties, i.e. interface Kapitza resistance coefficient $\bar{r}_{Q}^{0}$ and the sensitivity $\bar{s}_{0}$ will increase with damage evolving. The damage variable here is a function of the interface effective 
(undamaged) free energy $\bar{\Psi}^{0}$, which in turn depends on both the interface temperature $\bar{\Theta}$ and the interface deformation gradient $\overline{\boldsymbol{F}}$. Here no distinction between thermal and mechanical damage has been made for the sake of simplicity and the fact that such distinction has not yet been physically motivated.

In summary, the key contributions of this work are as follows:

- To derive the governing equations of a thermo-mechanical solid possessing thermally GI and mechanically coherent energetic interface subject to in-plane degradation, within the fully-nonlinear three dimensional setting.

- To present a thermodynamically consistent formulation and derive the dissipation inequality on the interface.

- To account for the effects of in-plane damage on thermo-mechanical properties of the interface.

- To derive the thermal and mechanical weak forms.

- To derive the consistent tangent stiffness matrices in the bulk and on the interface.

- To present details of the computation of solids possessing thermally GI and mechanically coherent energetic interfaces within the three-dimensional, non-linear and transient setting.

- To illustrate the theory with the help of numerical examples using the finite element method.

This manuscript is organized as follows. First the notation and certain key concepts are briefly introduced. Section 2 summarizes the kinematics of non-linear continuum mechanics. The local governing equations including the additional contributions from the interface, together with the constitutive relations are given in section 3 A numerical framework for the interface is established in section 4 . The framework includes the weak formulation of the governing equations, the corresponding finite element implementation and the derivation of the consistent stiffness matrices. A series of numerical examples, based on the finite element approximation of the weak form, is presented in section 5 to elucidate the theory. Section 6 concludes this work.

\section{Problem definition}

This section summarizes the kinematics of non-linear continuum mechanics including thermally general imperfect and mechanically coherent energetic interfaces and introduces the notation adopted here. Further details on the kinematics of deformable interfaces can be found in [30]. All over-lined quantities correspond to the interface円 Table 1 gathers a list of notations frequently used in this manuscript. Consider a continuum body $\mathscr{B}$ that takes the material

\footnotetext{
${ }^{1}$ Direct notation is adopted throughout. Occasional use is made of index notation, the summation convention for repeated indices being implied. The three-dimensional Euclidean space is denoted by $\mathbb{E}^{3}$. The scalar product of two vectors $\boldsymbol{a}$ and $\boldsymbol{b}$ is denoted by $\boldsymbol{a} \cdot \boldsymbol{b}=[\boldsymbol{a}]_{i}[\boldsymbol{b}]_{i}$. The scalar
} 
Table 1: List of important notations. Over-lined quantities $\{\overline{\boldsymbol{\bullet}}\}$ correspond to the interface.

\begin{tabular}{|c|c|c|c|}
\hline $\boldsymbol{F}$ & bulk material deformation gradient & $\bar{F}$ & interface material deformation gradient \\
\hline$\varphi$ & bulk deformation map & $\bar{\varphi}$ & interface deformation map \\
\hline$X$ & bulk material coordinates & $\bar{X}$ & interface material coordinates \\
\hline$x$ & bulk spatial coordinates & $\bar{x}$ & interface spatial coordinates \\
\hline$\Theta$ & bulk temperature & $\bar{\Theta}$ & interface temperature \\
\hline$\Theta_{0}$ & bulk initial temperature & $\bar{\Theta}_{0}$ & interface initial temperature \\
\hline$N$ & bulk material normal to surface & $\bar{N}$ & interface material normal to interface \\
\hline$n$ & bulk spatial normal to surface & $\bar{n}$ & interface spatial normal to interface \\
\hline$\Psi$ & bulk Helmholtz energy & $\bar{\Psi}$ & interface nominal Helmholtz energy \\
\hline$\Xi_{\mathrm{s}}$ & bulk specific entropy & $\bar{\Xi}_{\mathrm{s}}$ & interface specific entropy \\
\hline$\Xi$ & bulk entropy & $\bar{\Xi}$ & interface nominal entropy \\
\hline $\boldsymbol{P}$ & bulk Piola stress & $\bar{P}$ & interface nominal Piola stress \\
\hline \multicolumn{4}{|c|}{ only interface } \\
\hline$\varphi^{ \pm}$ & deformation maps of \pm side & $\boldsymbol{x}^{ \pm}$ & spatial coordinates of \pm side \\
\hline$\tilde{n}$ & spatial normal to interface boundary & $\widetilde{N}$ & material normal to interface boundary \\
\hline $\bar{\Psi}^{0}$ & undamaged Helmholtz energy & $\bar{D}_{\|}$ & damage variable \\
\hline$\overline{\boldsymbol{P}}_{0}$ & undamaged Piola stress tensor & $\bar{\Xi}^{0}$ & undamaged entropy \\
\hline $\bar{F}_{\text {loc }}$ & local equivalent distortion & $\bar{F}_{\text {nloc }}$ & non-local equivalent distortion \\
\hline $\bar{F}_{0}$ & elastic limit & $\bar{F}_{\max }$ & maximum attained $\bar{F}_{\text {nloc }}$ \\
\hline $\bar{s}_{0}$ & undamaged sensitivity & $\bar{r}_{Q}^{0}$ & undamaged Kapitza resistance coefficient \\
\hline
\end{tabular}

configuration $\mathcal{B}_{0} \subset \mathbb{E}^{3}$ at time $t=0$, and the spatial configuration $\mathcal{B}_{t}$ at $t>0$, as depicted in Fig. 1 . The body $\mathscr{B}$ is partitioned into two disjoint subdomains, $\mathcal{B}_{0}^{+}$and $\mathcal{B}_{0}^{-}$, by an interface $\mathcal{I}_{0}$, thus the bulk is defined by $\mathcal{B}_{0}:=\mathcal{B}_{0}^{+} \cup \mathcal{B}_{0}^{-}$, with reference placements of material particles labeled $\boldsymbol{X}$. The two sides of the interface $\mathcal{I}_{0}$ are denoted $\mathcal{I}_{0}^{+}:=\partial \mathcal{B}_{0}^{+} \cap \mathcal{I}_{0}$ and $\mathcal{I}^{-}:=\partial \mathcal{B}_{0}^{-} \cap \mathcal{I}_{0}$. The material particles on the interface are labeled $\overline{\boldsymbol{X}}$. The outward unit normal to $\partial \mathcal{B}_{0}$ is denoted $N$. The outward unit normal to the boundary of the interface $\partial \mathcal{I}_{0}$, tangent to the interface $\mathcal{I}_{0}$ is denoted $\widetilde{N}$. The unit normal to $\mathcal{I}_{0}$ is denoted $\bar{N}$ whose direction is conventionally taken to point from the negative side of the interface to the positive side. The spatial counterparts of the various unit normals are $\boldsymbol{n}, \widetilde{\boldsymbol{n}}$ and $\overline{\boldsymbol{n}}$, respectively. The deformation maps of the bulk, and the negative and positive sides of the interface are denoted $\varphi, \varphi^{-}$and $\varphi^{+}$, respectively. The restriction of the motion $\varphi$ to the interface is defined by $\bar{\varphi}$. The current placements of particles in the bulk and on the

product of two second-order tensors $\boldsymbol{A}$ and $\boldsymbol{B}$ is denoted by $\boldsymbol{A}: \boldsymbol{B}=[\boldsymbol{A}]_{i j}[\boldsymbol{B}]_{i j}$. The composition of two second-order tensors $\boldsymbol{A}$ and $\boldsymbol{B}$, denoted by $\boldsymbol{A} \cdot \boldsymbol{B}$, is a second-order tensor with coefficients $[\boldsymbol{A} \cdot \boldsymbol{B}]_{i j}=[\boldsymbol{A}]_{i m}[\boldsymbol{B}]_{m j}$. The non-standard products of a fourth-order tensor $\mathbb{C}$ and a vector $\boldsymbol{b}$ is defined by $[\boldsymbol{b}: \mathbb{C}]_{i k l}=[\mathbb{C}]_{i j k l}[\boldsymbol{b}]_{j}$. The action of a second-order tensor $\boldsymbol{A}$ on a vector $\boldsymbol{a}$ is given by $[\boldsymbol{A} \cdot \boldsymbol{a}]_{i}=[\boldsymbol{A}]_{i j}[\boldsymbol{a}]_{j}$. The standard product of a fourth-order tensor $\mathbb{C}$ and a second-order tensor $\boldsymbol{A}$ is defined by $[\mathbb{C}: \boldsymbol{A}]_{i j}=[\mathbb{C}]_{i j k l}[\boldsymbol{A}]_{k l}$. The dyadic product of two vectors $\boldsymbol{a}$ and $\boldsymbol{b}$ is a secondorder tensor $\boldsymbol{D}=\boldsymbol{a} \otimes \boldsymbol{b}$ with $[\boldsymbol{D}]_{i j}=[\boldsymbol{a}]_{i}[\boldsymbol{b}]_{j}$. Two non-standard dyadic products of two second-order tensors $\boldsymbol{A}$ and $\boldsymbol{B}$ are the fourth-order tensors $[\boldsymbol{A} \bar{\otimes} \boldsymbol{B}]_{i j k l}=[\boldsymbol{A}]_{i k}[\boldsymbol{B}]_{j l}$ and $[\boldsymbol{A} \otimes \boldsymbol{B}]_{i j k l}=[\boldsymbol{A}]_{i l}[\boldsymbol{B}]_{j k}$. The average and jump of a quantity $\{\bullet\}$ over an interface are defined by $\{\{\bullet\}\}=\frac{1}{2}\left[\{\bullet\}^{+}+\{\bullet\}^{-}\right]$ and $\llbracket\{\bullet\} \rrbracket=\{\bullet\}^{+}-\{\bullet\}^{-}$, respectively. 


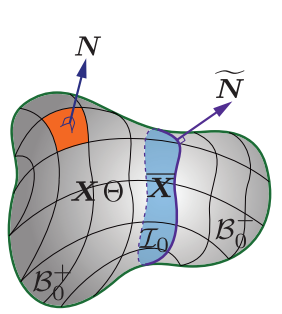

time $t=0$

material configuration

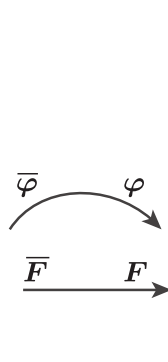

ion

Figure 2: The bulk domain $\mathcal{B}_{0}$, the bulk subdomains $\mathcal{B}_{0}^{ \pm}$, the interface $\mathcal{I}_{0}$, the two sides of the interface $\mathcal{I}_{0}^{ \pm}$and the unit normals to the surface $N$, the interface $\bar{N}$, and boundary of the interface $\widetilde{N}$, all defined in the material configuration. The bulk, interface and the two sides of interface deformation maps, denoted as $\varphi, \bar{\varphi}$ and $\varphi^{ \pm}$, respectively, map the material configuration to the spatial configuration at time $t$. The bulk domain $\mathcal{B}_{t}$, the bulk subdomains $\mathcal{B}_{t}^{ \pm}$, the interface $\mathcal{I}_{t}$ and its two sides $\mathcal{I}_{t}^{ \pm}$, the unit normals to the surface $\boldsymbol{n}$, interface $\overline{\boldsymbol{n}}$, and boundary of the interface $\widetilde{\boldsymbol{n}}$, all defined in the spatial configuration. The bulk temperatures on plus and minus side of the interface and the interface temperature are denoted by $\Theta^{+}, \Theta^{-}$and $\bar{\Theta}$, respectively. The interface unit normal is pointing from the negative side of the interface to the positive side. The bulk and (rank-deficient) interface deformation gradients are $\boldsymbol{F}$ and $\overline{\boldsymbol{F}}$, respectively. The interface is mechanically coherent and thermally non-coherent.

two sides of the interface are denoted $\boldsymbol{x}$ and $\boldsymbol{x}^{\mp}$ where the spatial placement of particles on the interface are designated as $\bar{x}$. One should note that $\varphi^{+}=\varphi^{-}=\bar{\varphi}$ and $x^{+}=x^{-}=\bar{x}$, for mechanically coherent interfaces. This means the interface placement is always between the two lateral sides of the interface. The interface and bulk temperature on two sides of the interface are denoted by $\bar{\Theta}, \Theta^{+}$and $\Theta^{-}$, respectively.

Remark 1 Since the interface is thermally general imperfect, the bulk temperatures $\Theta^{+}$and $\Theta^{-}$can differ from each other. This is in contrast with a highly-conductive interface where the jump of temperature across the interface vanishes and thus $\bar{\Theta}=\Theta^{+}=\Theta^{-}$. Moreover, on a thermally general imperfect interface, the relation between the bulk and the interface temperature $\bar{\Theta}$ is in general unknown. In other words, the interface temperature does not necessarily take a value between the bulk temperatures on the two sides of the interface [see 21, 28. for further details].

The bulk and the (rank-deficient) interface deformation gradients $\boldsymbol{F}$ and $\overline{\boldsymbol{F}}$, together with the corresponding velocities $\boldsymbol{V}$ and $\overline{\boldsymbol{V}}$ are, respectively, defined by

$$
\boldsymbol{F}(\boldsymbol{X}, t):=\operatorname{Grad} \varphi(\boldsymbol{X}, t) \quad, \quad \boldsymbol{V}:=\mathrm{D}_{t} \boldsymbol{\varphi}(\boldsymbol{X}, t) \quad \text { and } \quad \overline{\boldsymbol{F}}(\overline{\boldsymbol{X}}, t):=\overline{\operatorname{Grad}} \overline{\boldsymbol{\varphi}}(\overline{\boldsymbol{X}}, t) \quad, \quad \overline{\boldsymbol{V}}:=\mathrm{D}_{t} \overline{\boldsymbol{\varphi}}(\overline{\boldsymbol{X}}, t)
$$

Thereby the interface gradient and divergence operators, respectively, read

$$
\overline{\operatorname{Grad}}\{\bar{\bullet}\}:=\operatorname{Grad}\{\bar{\bullet}\} \cdot \overline{\boldsymbol{I}} \quad \text { and } \quad \overline{\operatorname{Div}}\{\bar{\bullet}\}:=\overline{\operatorname{Grad}}\{\bar{\bullet}\}: \overline{\boldsymbol{I}} \quad \text { with } \quad \overline{\boldsymbol{I}}:=\boldsymbol{I}-\overline{\boldsymbol{N}} \otimes \overline{\boldsymbol{N}}
$$

where $\overline{\boldsymbol{I}}$ and $\boldsymbol{I}$ denote the interface and bulk unit tensors. Their spatial counterparts are denoted $\overline{\boldsymbol{i}}$ and $\boldsymbol{i}$. Finally the bulk and interface Jacobians are denoted by $J:=\operatorname{det} \boldsymbol{F}>0$, and $\bar{J}:=\overline{\operatorname{det}} \overline{\boldsymbol{F}}>0$, respectively, with $\overline{\operatorname{det}}\{\bullet\} \operatorname{denoting} \operatorname{the}$ area determinant [44]. 
Table 2: Localized force, energy and entropy balances in the bulk and on the interface in the material configuration. The notation $\{\bullet\}^{\mathrm{P}}$ is to denote prescribed quantities.

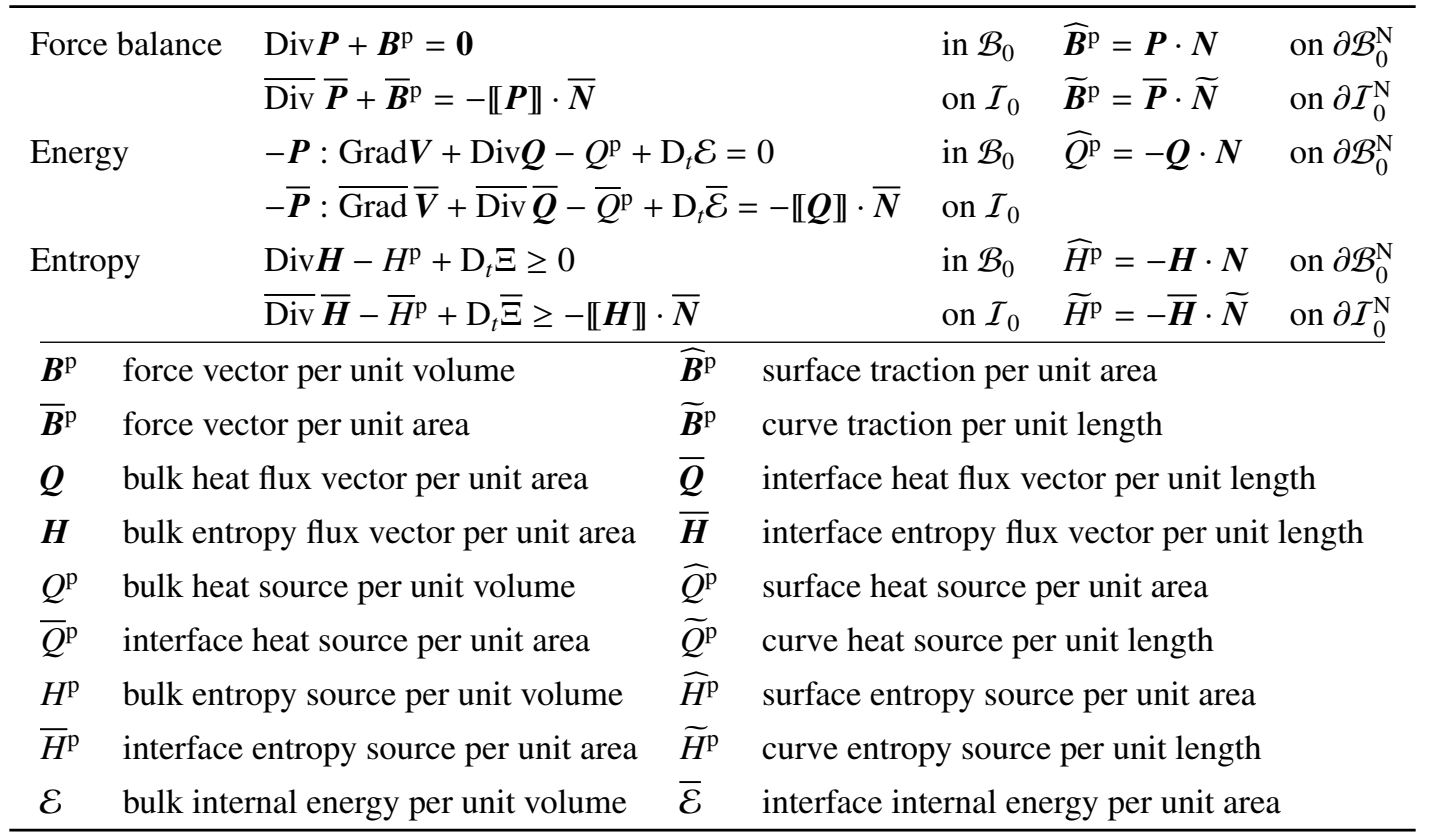

Balance of angular momentum results in the symmetry of the bulk Cauchy stress, i.e. $\boldsymbol{P} \cdot \boldsymbol{F}^{t}=\boldsymbol{F} \cdot \boldsymbol{P}^{t}$ and the interface Cauchy stress, i.e. $\overline{\boldsymbol{P}} \cdot \overline{\boldsymbol{F}}^{t}=\overline{\boldsymbol{F}} \cdot \overline{\boldsymbol{P}} t$ in the material configuration.

\section{Governing equations}

The local balance equations of force, energy and entropy in the bulk and on the interface together with the associated boundary conditions are listed in Table 2] [see 21, for further details]. The here considered interface model deals with interfaces that are

- mechanically coherent, $\llbracket \varphi \rrbracket=\mathbf{0}$,

- mechanically energetic, thus $\llbracket \boldsymbol{P} \rrbracket \cdot \overline{\boldsymbol{N}} \neq \mathbf{0}$ and

- thermally general imperfect, $\llbracket Q \rrbracket \cdot \bar{N} \neq 0$ and $\llbracket \Theta \rrbracket \neq 0$.

The third property, i.e. thermal general imperfection of the interface is characterized by allowing a jump both in the temperature and the normal heat flux, $\llbracket \Theta \rrbracket \neq 0$ and $\llbracket Q \rrbracket \cdot \bar{N} \neq 0$, respectively, across the interface. Also note that a thermally GI interface is fully dissipative. See section 3 for further elaborations.

Remark 2 In what follows we briefly discuss different kinds of thermal interfaces:

- a thermally perfect interface is recovered when $\llbracket Q \rrbracket \cdot \bar{N}=0$ and $\llbracket \Theta \rrbracket=0$; 
- a highly-conductive interface imposes a vanishing temperature jump across the interface, while allowing for the jump of normal heat flux across the interface, i.e. $\llbracket \Theta \rrbracket=0$ and $\llbracket Q \rrbracket \cdot \bar{N} \neq 0$. Note that a continuous temperature distribution across the interface does not necessary imply a HC interface. See [28] for further details. Furthermore, an HC interface is non-dissipative due to the vanishing temperature jump across the interface, which results in the interface temperature to be identical to the bulk temperatures on the two sides of the interface;

- a lowly-conductive (LC) interface allows for a temperature jump but not for a jump in the normal heat flux across the interface, i.e. $\llbracket \Theta \rrbracket \neq 0$ and $\llbracket Q \rrbracket \cdot \bar{N}=0$. This model is subject to Kapitza's assumption of thermal resistance. Note that a LC interface is semi-dissipative (possessing only one dissipation contribution, see section 3 for further discussions). For this interface a connection between the interface and the bulk temperature, in general, can not be drawn;

- a semi-dissipative (SD) interface is a generalization of the LC interface so that the jump in both the temperature and the normal heat flux is admissible, i.e. $\llbracket Q \rrbracket \cdot \bar{N} \neq 0$ and $\llbracket \Theta \rrbracket \neq 0$. Analogous to the LC interface, the same dissipation contribution is non-vanishing for a SD interface. However, unlike a LC interface, the SD interface imposes a relation between the interface and the bulk temperature, or more precisely between what we call interface and bulk coldness. The coldness here is defined as the inverse of the temperature;

- a fully-dissipative (FD) interface is a GI interface, similar to a SD interface in the sense that both $Q Q \rrbracket \cdot$ $\bar{N} \neq 0$ and $\llbracket \Theta \rrbracket \neq 0$ are admissible. Nonetheless, a FD interface is generalized to possess two dissipation contributions. Consequently, a relation in general between interface and bulk temperature cannot be established (analogously to an LC interface). Additionally, for a FD interface the interface temperature shall be considered as an independent degree of freedom.

Furthermore, both thermal and mechanical properties of the interface are affected by the interface in-plane degradation. In doing so a reduction factor $\left[1-\bar{D}_{\|}\right]$is introduced, which reduces the mechanical and in-plane thermal properties of the interface as the damage $\bar{D}_{\|}$evolves. The out-of-plane thermal properties of the interface, the sensitivity $\bar{s}_{0}$ and the Kapitza thermal resistance $\bar{r}_{Q}^{0}$, are inversely affected by the reduction factor, i.e. $\bar{s}=\bar{s}_{0} /\left[1-\bar{D}_{\|}\right]$and $\bar{r}_{Q}=\bar{r}_{Q}^{0} /\left[1-\bar{D}_{\|}\right]$. Note that in this work the damage variable is a function of the non-local equivalent distortion $\bar{F}_{\text {nloc }}$, which in turn depends on the interface deformation gradient $\overline{\boldsymbol{F}}$ and temperature $\overline{\boldsymbol{\Theta}}$. The interface Piola stress $\overline{\boldsymbol{P}}$ is a superficial tensor field possessing the property $\overline{\boldsymbol{P}} \cdot \overline{\boldsymbol{N}}=\mathbf{0}$. It is noteworthy to mention that the interface is mechanically coherent and due to the interface energetics, a discontinuity in the traction across the interface is allowed and hence $\llbracket P \rrbracket \cdot \bar{N} \neq \mathbf{0}$.

Next the bulk and interface free energies, the corresponding constitutive relations and temperature evolution equations are give in Table 3 Note that $0 \leq \bar{D}_{\|}(\overline{\boldsymbol{F}}, \bar{\Theta}) \leq 1, \bar{\vartheta}$ is an internal variable, $\boldsymbol{k}$ and $\overline{\boldsymbol{k}}_{0}$ denote the bulk and interface positive (semi-)definite thermal conductivity tensors. For thermally isotropic materials in the spatial configuration, $\boldsymbol{k}=k \boldsymbol{i}$ and $\overline{\boldsymbol{k}}_{0}=\bar{k}_{0} \overline{\boldsymbol{i}}$, where the scalars $k \geq 0$ and $\bar{k}_{0} \geq 0$ are the thermal conductivity coefficients in the bulk and on the interface, respectively. The heat capacity coefficients in the bulk and on the interface are denoted by $c_{\boldsymbol{F}}$ and $\bar{c}_{\overline{\boldsymbol{F}}}=\left[1-\bar{D}_{\|}\right] \bar{c}_{\bar{F}}^{0}$, where $\bar{c}_{\bar{F}}^{0}$ is the interface heat capacity coefficient associated with the undamaged (virgin) state of the interface material. 
Table 3: Bulk and interface free energies, the corresponding constitutive relations and temperature evolution equations.

\begin{tabular}{|c|c|c|}
\hline \multirow[t]{2}{*}{ Free energy } & $\Psi \equiv \Psi(\boldsymbol{F}, \Theta)$ & in $\mathcal{B}_{0}$ \\
\hline & $\bar{\Psi} \equiv \bar{\Psi}\left(\overline{\boldsymbol{F}}, \bar{\Theta}, \bar{D}_{\|}, \bar{\vartheta}\right)=\left[1-\bar{D}_{\|}\right] \bar{\Psi}^{0}$ & on $\mathcal{I}_{0}$ \\
\hline \multirow[t]{4}{*}{ Constitutive relations } & $\boldsymbol{P}:=\partial \Psi / \partial \boldsymbol{F}$ and $\Xi:=-\partial \Psi / \partial \Theta$ & in $\mathcal{B}_{0}$ \\
\hline & $\overline{\boldsymbol{P}}:=\partial \bar{\Psi} / \partial \overline{\boldsymbol{F}}=\left[1-\bar{D}_{\|}\right] \overline{\boldsymbol{P}}_{0}$ and $\bar{\Xi}:=-\partial \bar{\Psi} / \partial \bar{\Theta}=\left[1-\bar{D}_{\|}\right] \bar{\Xi}^{0}$ & on $\mathcal{I}_{0}$ \\
\hline & $\boldsymbol{Q}=-J \boldsymbol{F}^{-1} \cdot \boldsymbol{k} \cdot \boldsymbol{F}^{-\mathrm{t}} \cdot \operatorname{Grad} \Theta$ & in $\mathcal{B}_{0}$ \\
\hline & $\overline{\boldsymbol{Q}}=-\bar{J} \overline{\boldsymbol{F}}^{-1} \cdot\left[1-\bar{D}_{\|}\right] \overline{\boldsymbol{k}}_{0} \cdot \overline{\boldsymbol{F}}^{-\mathrm{t}} \cdot \overline{\mathrm{Grad}} \overline{\boldsymbol{\Theta}}$ & on $\mathcal{I}_{0}$ \\
\hline \multirow[t]{2}{*}{ Temperature evolution } & $c_{\boldsymbol{F}} \mathrm{D}_{t} \Theta=-\operatorname{Div} \boldsymbol{Q}+\Theta \partial_{\Theta} \boldsymbol{P}: \mathrm{D}_{t} \boldsymbol{F}+Q^{\mathrm{p}}$ with $c_{\boldsymbol{F}}:=-\Theta \frac{\partial^{2} \Psi}{\partial \Theta^{2}}$ & in $\mathcal{B}_{0}^{\mathrm{N}}$ \\
\hline & $\bar{c}_{\overline{\boldsymbol{F}}} \mathrm{D}_{t} \overline{\boldsymbol{\Theta}}=-\overline{\mathrm{Div}} \overline{\boldsymbol{Q}}+\overline{\boldsymbol{\Theta}} \partial_{\bar{\Theta}} \overline{\boldsymbol{P}}: \mathrm{D}_{t} \overline{\boldsymbol{F}}+\bar{Q}^{\mathrm{p}}-\llbracket \boldsymbol{Q} \rrbracket \cdot \overline{\boldsymbol{N}}$ with $\bar{c}_{\overline{\boldsymbol{F}}}:=-\left[1-\bar{D}_{\|}\right] \overline{\boldsymbol{\Theta}} \frac{\partial^{2} \Psi^{0}}{\partial \bar{\Theta}^{2}}$ & on $I_{0}^{\mathrm{N}}$ \\
\hline
\end{tabular}

To proceed a Helmholtz energy $\sqrt{2}$ is considered for the interface containing the following arguments [21]

$$
\bar{\Psi}(\overline{\boldsymbol{F}}, \bar{\Theta}, \bar{D}, \bar{\vartheta})=[1-\bar{D}] \bar{\Psi}_{0}(\overline{\boldsymbol{F}}, \bar{\Theta})+\int_{0}^{\bar{\vartheta}} \overline{\mathcal{H}}\left(\bar{\vartheta}^{*}\right) \mathrm{d} \bar{\vartheta}^{*},
$$

where $\overline{\mathcal{H}}(\bar{\vartheta})$ denotes a monotonically increasing function depending on the internal variable $\bar{\vartheta}$. Now by differentiating Eq. (3) with respect to time, particularizing the Clausius-Plank inequality and making use of the constitutive relations, one expresses the interface reduced dissipation $\overline{\mathcal{D}}_{\text {red }}$ as [see 21, for further details]

$$
\overline{\mathcal{D}}_{\text {red }}=\underbrace{\bar{Y} \dot{\bar{D}}_{\|}-\overline{\mathcal{H}}(\bar{\vartheta}) \dot{\bar{\vartheta}}}_{\overline{\mathcal{D}}_{\|}}+\underbrace{\left.\bar{\Theta}\left[\llbracket \Theta^{-1} \rrbracket \llbracket Q \boldsymbol{Q}\right\}-\left[\bar{\Theta}^{-1}-\left\{\Theta^{-1}\right\}\right] \llbracket \boldsymbol{Q} \rrbracket\right] \cdot \overline{\boldsymbol{N}}}_{\overline{\mathcal{D}}_{\sharp}} \geq 0 \quad \text { with } \quad \bar{Y}=\bar{\Psi}^{0}(\overline{\boldsymbol{F}}, \overline{\boldsymbol{\Theta}})=-\frac{\partial \bar{\Psi}}{\partial \bar{D}_{\|}},
$$

where the quantity $\bar{Y}$ is the thermodynamic force conjugate to the interface damage variable $\bar{D}_{\|}$. Next, together with satisfying $\overline{\mathcal{D}}_{\|} \geq 0$ a damage condition $\bar{\Upsilon}$ is introduced as [43]

$$
\bar{\Upsilon}(\bar{Y}, \overline{\mathcal{H}})=\bar{v}(\bar{Y})-\overline{\mathcal{H}}(\bar{\vartheta}) \leq 0
$$

with $\bar{v}$ being a monotonically increasing function. The damage evolution law and the Kuhn-Tucker conditions can be obtained from the postulate of maximum dissipation using the Lagrange-multiplier method. Now by choosing $\bar{v}(\bullet)=$ $\overline{\mathcal{H}}(\bullet)$, and defining the change of variables $\bar{F}_{\text {max }}:=f(\bar{\vartheta})$ and $\bar{F}_{\text {nloc }}:=f(\bar{Y})$ and assuming $f$ to be a monotonically increasing function with the property $f(0)=0$, an alternative damage condition to Eq. (5) takes the form extended to

\footnotetext{
${ }^{2}$ The integral term in Eq. 33 is introduced in analogy with that of Simo and Hughes [41 section. 1.3.3] and is the energy storage in the material due to the accumulation of microscopic defects.
} 
integral-type non-locality [21]

$$
\bar{\phi}\left(\bar{F}_{\text {nloc }}, \bar{F}_{\text {max }}\right)=\bar{F}_{\text {nloc }}-\bar{F}_{\text {max }} \leq 0 \quad \text { with } \quad \bar{F}_{\text {nloc }}\left(\overline{\boldsymbol{x}}_{\mathrm{r}}\right)=\int_{I_{0}} \bar{\omega}\left(\overline{\boldsymbol{x}}_{\mathrm{r}}, \overline{\boldsymbol{x}}_{\mathrm{s}}\right) \bar{F}_{\text {loc }}\left(\boldsymbol{x}_{s}\right) \mathrm{d} A \quad \text { and } \quad \bar{F}_{\text {loc }}:=\sqrt{\frac{2 \bar{Y}}{\bar{E}}},
$$

where $\bar{F}_{\max }(t)=\max _{s \in[0, t]}\left\{\bar{F}_{0},\left.\bar{F}_{\text {nloc }}\right|_{s}\right\}, \bar{F}_{0}$ is the damage threshold, $\bar{F}_{\text {loc }}$ is the local equivalent distortion, and $\bar{E}$ is the interface Young's modulus. Note that the damage variable is eventually simply a function of $\bar{F}_{\text {max }}$, i.e. $\bar{D}_{\|}=\overline{\mathscr{D}}_{\|}\left(\bar{F}_{\max }\right)$. In Eq. $[6]_{2}, \bar{\omega}\left(\overline{\boldsymbol{x}}_{\mathrm{r}}, \overline{\boldsymbol{x}}_{\mathrm{s}}\right)$ is a given non-local weight function depending on the geodesic distance $\bar{r}=\left\|\overline{\boldsymbol{x}}_{\mathrm{r}}-\overline{\boldsymbol{x}}_{\mathrm{s}}\right\|_{I}$ between the source point $\overline{\boldsymbol{x}}_{\mathrm{s}}$ and the receiver point $\overline{\boldsymbol{x}}_{\mathrm{r}}$. On the interface the weight function $\bar{\omega}$ here is defined as

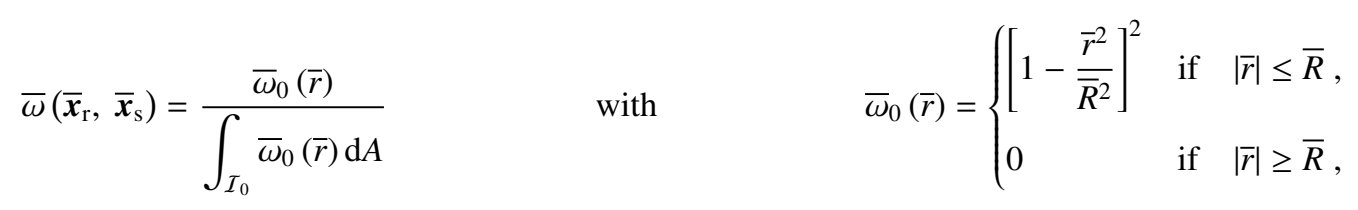

where $\bar{\omega}_{0}(\bar{r})$ is a non-negative and monotonically decreasing (for $\bar{r} \geq 0$ ) piecewise polynomial bell-shaped function. The interface interaction radius is denoted by $\bar{R}$. The damage function, relating $\bar{D}_{\|}$to the history variable $\bar{F}_{\text {max }}$ is given

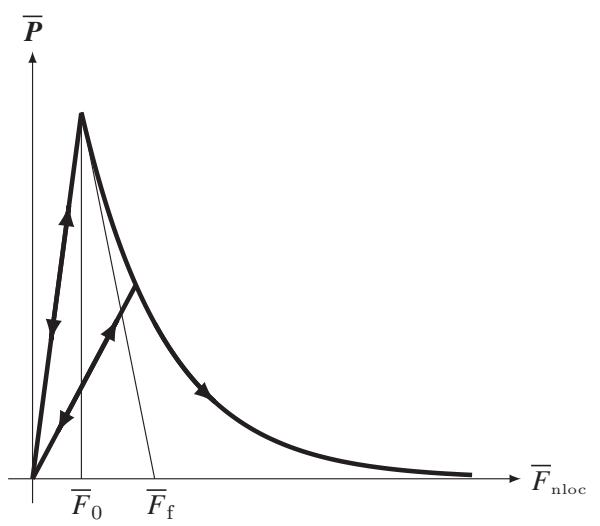

(a)

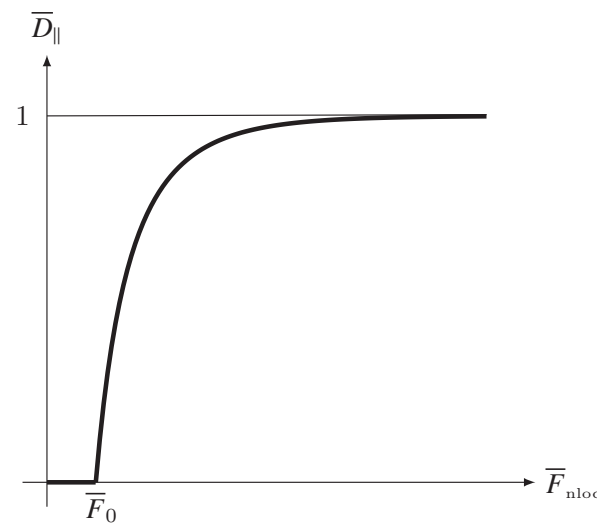

(b)

Figure 3: Stress vs. non-local equivalent distortion with exponential softening on the interface (a). Damage variable vs. non-local equivalent distortion associated with Fig. 8 (b). The parameters $\bar{F}_{0}$ and $\bar{F}_{\mathrm{f}}$ are the interface critical equivalent distortion and ductility response.

as follows (see Fig. 3 (b)):

$$
\bar{D}_{\|}=\overline{\mathscr{D}}_{\|}\left(\bar{F}_{\max }\right)=\left\{\begin{array}{lll}
0 & \text { if } & \bar{F}_{\max } \leq \bar{F}_{0} \\
1-\frac{\bar{F}_{0}}{\bar{F}_{\max }} \exp \left(-\frac{\bar{F}_{\max }-\bar{F}_{0}}{\bar{F}_{\mathrm{f}}-\bar{F}_{0}}\right) & \text { if } & \bar{F}_{\max } \geq \bar{F}_{0},
\end{array}\right.
$$

where $\bar{F}_{\mathrm{f}}$ affects the ductility of the response. An illustration is depicted in Fig. 3 .a). 
To satisfy $\overline{\mathcal{D}}_{\nVdash} \geq 0$ in Eq. $[4\}_{1}$, using the relation $\llbracket \Theta^{-1} \rrbracket=-\llbracket \Theta \rrbracket\left\{\Theta^{-1}\right\}\{\Theta\}^{-1}$, we enforce the fulfillment of the following two conditions:

$$
{ }^{1} \overline{\mathcal{D}}_{\nVdash}=-\llbracket \Theta \rrbracket\{Q\} \cdot \overline{\boldsymbol{N}} \geq 0 \quad \text { and } \quad{ }^{2} \overline{\mathcal{D}}_{\nVdash}=-\left[\bar{\Theta}^{-1}-\left\{\Theta^{-1}\right\}\right] \llbracket Q \rrbracket \cdot \overline{\boldsymbol{N}} \geq 0
$$

Remark 3 For the interface considered here both dissipation contributions in (9) are positive and hence the interface is termed fully dissipative. For a HC interface both of these dissipation contributions vanish since $\llbracket \Theta \rrbracket=0$. Both $S D$ and $L C$ interface allow for ${ }^{1} \overline{\mathcal{D}}_{\Varangle}$ to be nonzero since $\llbracket \Theta \rrbracket \neq 0$. The difference however is that for a SD interface $\llbracket Q \rrbracket \cdot \overline{\boldsymbol{N}} \neq 0$ and $\left[\bar{\Theta}^{-1}-\left\{\Theta^{-1}\right\}\right]=0$, whereas for an LC interface $\llbracket Q \rrbracket \cdot \overline{\boldsymbol{N}}=0$ and a relation between the interface and the bulk temperatures is in general unknown (see also Remark 2).

To this end, Fourier-like relations are introduced as follows:

$$
\llbracket \Theta \rrbracket=-\frac{\bar{r}_{Q}^{0}}{\left[1-\bar{D}_{\|}\right]}\{Q\} \cdot \bar{N} \quad \text { and } \quad \bar{\Theta}^{-1}-\left\{\Theta^{-1}\right\}=-\frac{\bar{s}_{0}}{\left[1-\bar{D}_{\|}\right]} \llbracket Q \rrbracket \cdot \bar{N},
$$

where $\bar{r}_{Q}^{0} \geq 0$ and $\bar{s}_{0} \geq 0$ are the undamaged (virgin) Kapitza resistance coefficient and the thermal sensitivity, respectively. As the interface damage grows and thus the reduction factor $\left[1-D_{\|}\right]$decreases, one expects a more pronounced jump in the temperature across the interface and a less strong coupling between the bulk and the interface temperatures due to $\bar{r}_{Q}^{0} /\left[1-\bar{D}_{\|}\right]$and $\bar{s}_{0} /\left[1-\bar{D}_{\|}\right]$taking higher values. This observation is illustrated by the numerical results which will be presented later.

\section{Computational framework}

In this section we establish a numerical framework that encompasses thermo-hyperelasticity combined with a nonlocal damage model on the thermally general imperfect and mechanically coherent energetic interface. The weak form, together with its temporal and spatial discretizations will be presented next. The localized force balance equations in the bulk and on the interface given in Table 2 are tested with vector valued test functions $\delta \varphi \in \mathscr{H}^{1}\left(\mathcal{B}_{0}\right)$ and $\delta \bar{\varphi} \in \mathscr{H}^{1}\left(\mathcal{I}_{0}\right)$, respectively. By integrating the result over all domains in the material configuration, using the bulk and interface divergence theorems and the superficiality properties of the interface Piola stress, the weak form of the balance of linear momentum reads

$$
\begin{aligned}
& \int_{\mathcal{B}_{0}} \boldsymbol{P}: \operatorname{Grad} \delta \boldsymbol{\varphi} \mathrm{d} V+\int_{\mathcal{I}_{0}}\left[1-\bar{D}_{\|}\right] \overline{\boldsymbol{P}}_{0}: \overline{\operatorname{Grad}} \delta \overline{\boldsymbol{\varphi}} \mathrm{d} A \\
& -\int_{\mathcal{B}_{0}} \delta \boldsymbol{\varphi} \cdot \boldsymbol{B}^{\mathrm{p}} \mathrm{d} V-\int_{\mathcal{I}_{0}} \delta \overline{\boldsymbol{\varphi}} \cdot \overline{\boldsymbol{B}}^{\mathrm{p}} \mathrm{d} A-\int_{\partial \mathcal{B}_{0}^{\mathrm{N}}} \delta \boldsymbol{\varphi} \cdot \widehat{\boldsymbol{B}}_{\mathrm{N}}^{\mathrm{p}} \mathrm{d} A-\int_{\partial I_{0}^{\mathrm{N}}} \delta \overline{\boldsymbol{\varphi}} \cdot \widetilde{\boldsymbol{B}}_{\mathrm{N}}^{\mathrm{p}} \mathrm{d} L=0, \\
& \forall \delta \boldsymbol{\varphi} \in \mathscr{H}^{1}\left(\mathcal{B}_{0}\right), \quad \forall \delta \overline{\boldsymbol{\varphi}} \in \mathscr{H}^{1}\left(\mathcal{I}_{0}\right) \quad \text { with } \quad \delta \overline{\boldsymbol{\varphi}}=\left\{\delta \boldsymbol{\varphi} \|\left.\right|_{\mathcal{I}_{0}} \quad \text { and } \quad \llbracket \delta \boldsymbol{\varphi} \rrbracket=\mathbf{0} .\right.
\end{aligned}
$$

Analogously the thermal weak form is derived first by testing the local temperature evolutions (see Table 3 in the bulk and on the interface with the scalar-valued test function $\delta \Theta \in \mathscr{H}_{0}^{1}\left(\mathcal{B}_{0}\right)$ and $\delta \bar{\Theta} \in \mathscr{H}_{0}^{1}\left(\mathcal{I}_{0}\right)$, respectively . The 
result is then integrated over the corresponding domains in the material configuration resulting in the global weak form of the temperature evolution equation as follows:

$$
\begin{aligned}
& \int_{\mathcal{B}_{0}} \boldsymbol{Q} \cdot \operatorname{Grad} \delta \Theta-\delta \Theta c_{\boldsymbol{F}} \mathrm{D}_{t} \Theta+\delta \Theta Q^{\mathrm{p}}+\delta \Theta \Theta \partial_{\Theta} \boldsymbol{P}: \mathrm{D}_{t} \boldsymbol{F} \mathrm{d} V+\int_{\partial \mathcal{B}_{0}^{\mathrm{N}}} \delta \Theta \widehat{Q}_{\mathrm{N}}^{\mathrm{p}} \mathrm{d} A \\
& +\int_{\bar{I}_{0}}\left[1-\bar{D}_{\|}\right] \overline{\boldsymbol{Q}}_{0} \cdot \overline{\operatorname{Grad}} \delta \bar{\Theta}-\delta \bar{\Theta}\left[1-\bar{D}_{\|}\right] \bar{c}_{\overline{\boldsymbol{F}}}^{0} \mathrm{D}_{t} \bar{\Theta}+\delta \overline{\Theta Q} \bar{Q}^{\mathrm{p}}+\delta \bar{\Theta} \bar{\Theta}\left[\left[1-\bar{D}_{\|}\right] \partial_{\bar{\Theta}} \overline{\boldsymbol{P}}_{0}-\partial_{\bar{\Theta}} \overline{\mathscr{D}}_{\|} \overline{\boldsymbol{P}}_{0}\right]: \mathrm{D}_{t} \overline{\boldsymbol{F}} \mathrm{d} A \\
& -\int_{\bar{I}_{0}} \llbracket \delta \Theta \|\left[1-\bar{D}_{\|}\right] \frac{1}{\bar{r}_{Q}^{0}} \llbracket \Theta \rrbracket-[\delta \bar{\Theta}-\{\delta \delta \Theta\}]\left[1-\bar{D}_{\|}\right] \frac{1}{\bar{S}_{0}}\left[\bar{\Theta}^{-1}-\left\{\Theta^{-1}\right\}\right] \mathrm{d} A=0 \\
& \forall \delta \Theta \in \mathscr{H}^{1}\left(\mathcal{B}_{0}\right) \text { and } \forall \delta \bar{\Theta} \in \mathscr{H}^{1}\left(\mathcal{I}_{0}\right),
\end{aligned}
$$

where $\overline{\boldsymbol{Q}}_{0}=-\bar{J} \overline{\boldsymbol{F}}^{-1} \cdot \overline{\boldsymbol{k}}_{0} \cdot \overline{\boldsymbol{F}}^{-\mathrm{t}} \cdot \overline{\mathrm{Grad}} \overline{\boldsymbol{\Theta}}$, is the undamaged heat conduction along the interface.

It is of great importance to mention that the current model can be simplified into other interface models. By setting the damage variable to zero, the model in [33] is retrieved, where a non-degrading thermally GI and mechanically coherent energetic interface is studied. A degrading HC interface model is obtained as in $[21]$ by setting $\llbracket \Theta \rrbracket=0$, and consequently $\{\{\Theta\}=\bar{\Theta}$, which then results in the last integral in Eq. 12 to vanish. A degrading LC interface can be modeled as in [20] by removing the second term in the last integral, due to the fact that the jump of normal heat flux across interface vanishes (see relation $10_{2}$ ). The finite element implementation is now given in Appendix A

\section{Numerical examples}

In this section we study the computational aspects of thermally GI and mechanically coherent energetic interfaces subject to in-plane degradation and their effects on the overall response of the body. The in- and out-of-plane thermomechanical response of the interface is affected by the interface in-plane degradation. In particular, we focus on the influence of interface damage on the out-of-plane thermal properties of the interface, i.e. $\bar{s}_{0}$ and $\bar{r}_{Q}^{0}$. It is important to point out that the solution procedure is robust and shows the asymptotically quadratic rate of convergence associated with the Newton-Raphson scheme. The computational domain is discretized using 1600 trilinear hexahedral elements.

The reversible material behavior in the bulk and on the interface is characterized by a thermo-hyperelastic Helmholtz energy functions. The damage affects the interface response by reducing the interface stiffness, heat capacity and heat conduction coefficient, and increasing the Kapitza coefficient $\bar{r}_{Q}^{0}$ and thermal sensitivity $\bar{s}_{0}$. Table B.5 in Appendix B gathers the effective (undamaged) Helmholtz energy functions together with their corresponding derivatives both in the bulk and on the interface. The corresponding material parameters for the bulk and interface are given in Table 4

Consider now the strip shown in Fig. 4 where a constant displacement is prescribed at the two opposite faces. The strip is partitioned into two homogeneous domains by an interface. The width and the thickness of the strip are kept constant. The thermal boundary condition is globally adiabatic i.e $\widehat{\boldsymbol{Q}}^{\mathrm{p}}=\widetilde{\boldsymbol{Q}}^{\mathrm{p}}=\mathbf{0}$. The thermal initial condition is a 
Table 4: Material properties assumed in the numerical examples.

\begin{tabular}{|c|c|c|c|c|}
\hline & bulk & & interface & \\
\hline Lamé constant & $\mu$ & $80193.8 \mathrm{~N} / \mathrm{mm}^{2}$ & $\bar{\mu}$ & $2 \times 80193.8 \mathrm{~N} / \mathrm{mm}$ \\
\hline Lamé constant & $\lambda$ & $110743.5 \mathrm{~N} / \mathrm{mm}^{2}$ & $\bar{\lambda}$ & $2 \times 110743.5 \mathrm{~N} / \mathrm{mm}$ \\
\hline compression modulus & $\kappa$ & $164206.03 \mathrm{~N} / \mathrm{mm}^{2}$ & $\bar{\kappa}$ & $2 \times 190937.3 \mathrm{~N} / \mathrm{mm}$ \\
\hline specific heat capacity & $c_{F}$ & $3.588 \mathrm{~N} /\left[\mathrm{mm}^{2} \mathrm{~K}\right]$ & $\bar{c}_{\boldsymbol{F}}^{0}$ & $3.588 \mathrm{~N} /[\mathrm{mmK}]$ \\
\hline heat conduction coeff. & $k$ & $45 \mathrm{~N} /[\mathrm{sK}]$ & $\bar{k}_{0}$ & $100 \times 45 \mathrm{Nmm} /[\mathrm{sK}]$ \\
\hline heat expansion coeff. & $\alpha$ & $10^{-5} 1 / \mathrm{K}$ & $\bar{\alpha}$ & {$[0-1.5] \times 10^{-5} 1 / \mathrm{K}$} \\
\hline initial temperature & $\Theta_{0}$ & $298 \mathrm{~K}$ & $\bar{\Theta}_{0}$ & $298 \mathrm{~K}$ \\
\hline \multicolumn{5}{|c|}{ only interface } \\
\hline & $\bar{F}_{0}$ & 0.005 & $\bar{F}_{\mathrm{f}}$ & 0.1 \\
\hline thermal resistance & $\bar{r}_{Q}^{0}$ & $0.1 \mathrm{~mm} \mathrm{sK} / \mathrm{N}$ & $\bar{R}$ & $0.1 \mathrm{~mm}$ \\
\hline
\end{tabular}

Note that $\kappa=\lambda+2 / 3 \mu$ and $\bar{\kappa}=\bar{\lambda}+\bar{\mu}$.

(a)

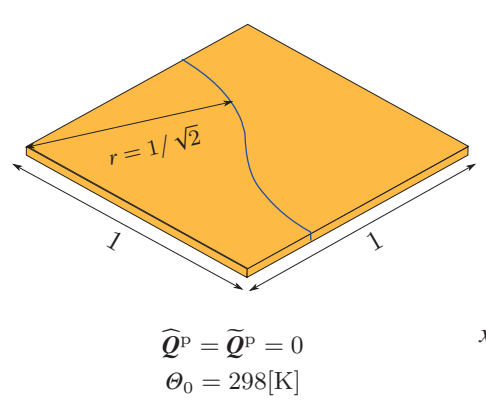

(b)

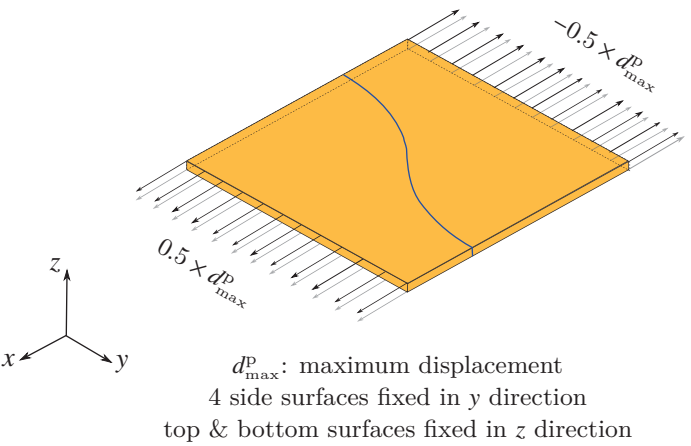

Figure 4: Strip with curved interface, (a) geometry and (b) applied boundary conditions. Dimensions are in mm. The thickness is 0.05 .

uniformly distributed temperature $\Theta_{0}=298 \mathrm{~K}$. In order to better understand the influence of a thermo-mechanical GI interface on the overall response of the body, all thermo-mechanical properties of the bulk are fixed. Similar examples of intact (non-degrading) LC, HC and GI interfaces can be found respectively in [29, 30, 33]. Degrading HC and LC interfaces are studied in the recent contributions [20, 21], respectively. It is mentioned that to obtain an HC interface behavior from the current model, one can assign infinitesimal values to $\bar{s}_{0}$ and $\bar{r}_{Q}^{0}$, which causes both dissipation contributions in 9 to vanish. An LC interface is obtained by setting only $\bar{s}_{0} \approx 0$, while $\bar{r}_{Q}^{0}$ is assigned a finite value. Note that we use Fourier-like relations $(10)$ to fulfill the inequalities in $(9)$.

In the first example we focus on the conductivity of a degrading interface. The domain is stretched up to $100 \%$ of its initial length in 40 equal steps where the total time is $10 \mathrm{~ms}$. Note that for this example $c_{\bar{F}}^{0}=\bar{\alpha}=0, \bar{k}_{0} / k=100$ 


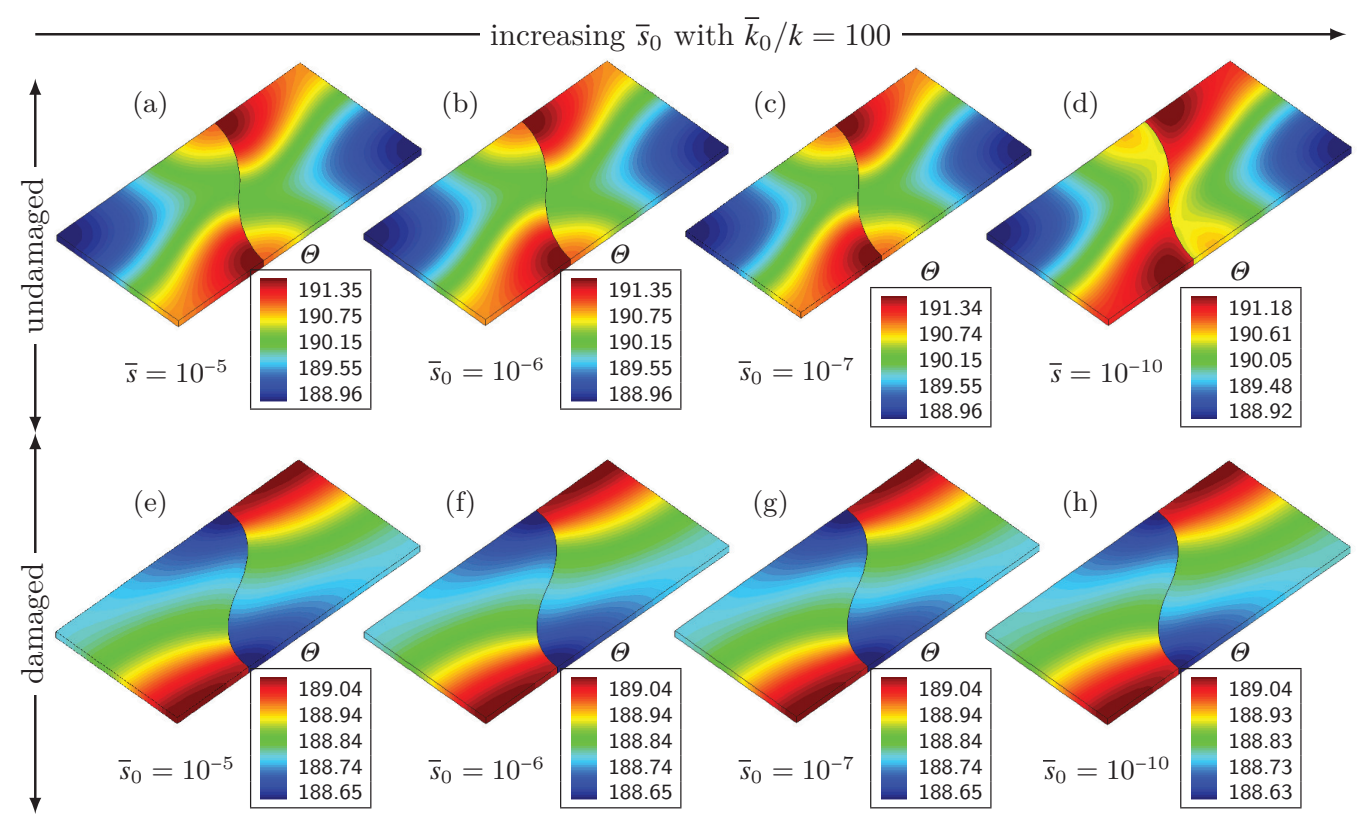

Figure 5: The bulk temperature distribution of the strip stretched up to $100 \%$ of its original length for $\bar{\mu} / \mu=\bar{\lambda} / \lambda=2 \mathrm{~mm}, \bar{r}_{Q}^{0}=0.1$ and $\bar{k}_{0} / k=100 \mathrm{~mm}$. Results (a)-(d) correspond to the intact interface, whereas results (e)-(h) correspond to the degrading interface.

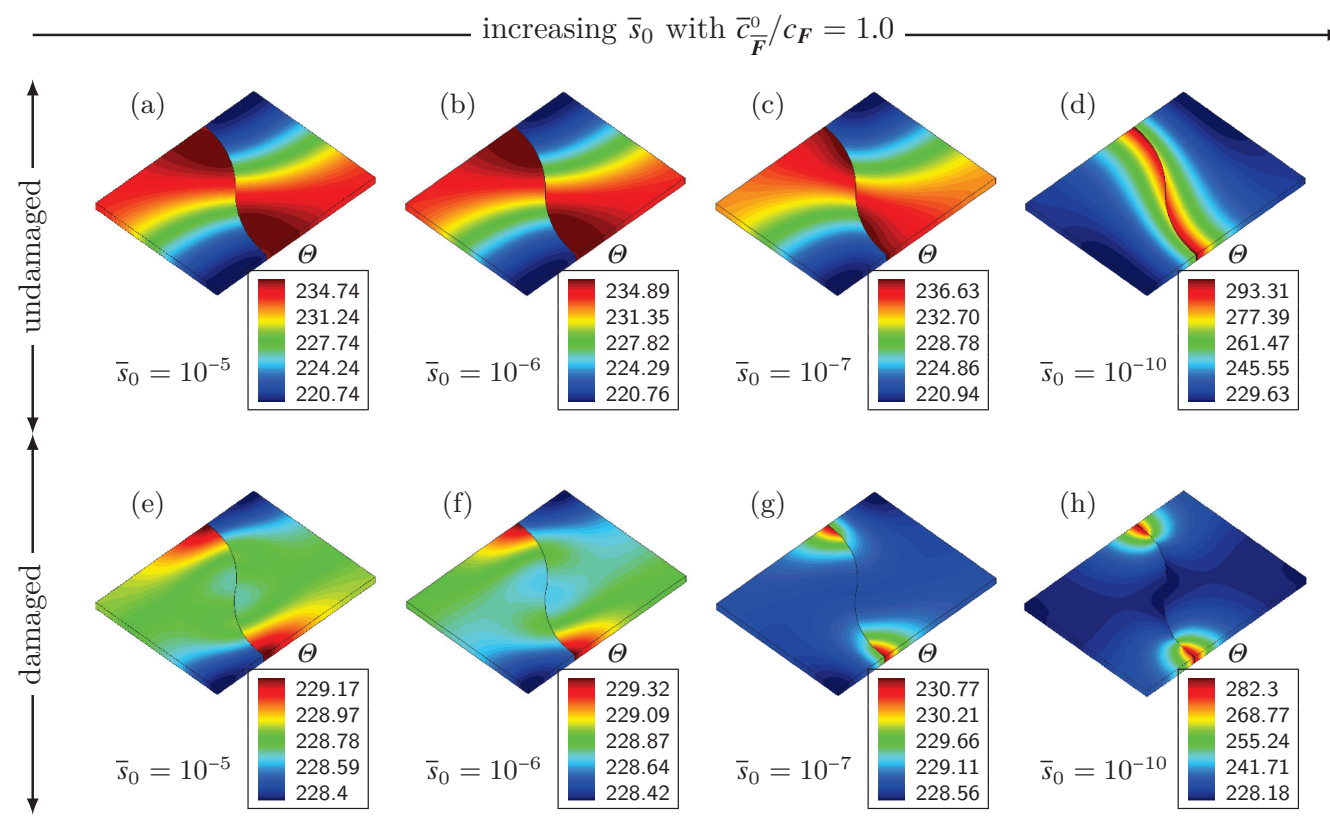

Figure 6: The bulk temperature distribution of the strip stretched up to $30 \%$ of its original length for $\bar{\mu} / \mu=\bar{\lambda} / \lambda=2 \mathrm{~mm}, \bar{r}_{Q}^{0}=0.1$ and $c_{\overline{\boldsymbol{F}}}^{0} / c_{\boldsymbol{F}}=$ $1 \mathrm{~mm}$. Results (a)-(d) correspond to the intact interface, whereas results (e)-(h) correspond to the degrading interface.

$\mathrm{mm}, \bar{r}_{Q}^{0}=0.1$, and $\bar{s}_{0}$ varies from $10^{-5}$ to $10^{-10}$. The results of two cases, undamaged and damaged interface, are compared and depicted in Fig. 5. It is observed that the temperature distribution along the intact interface is more 


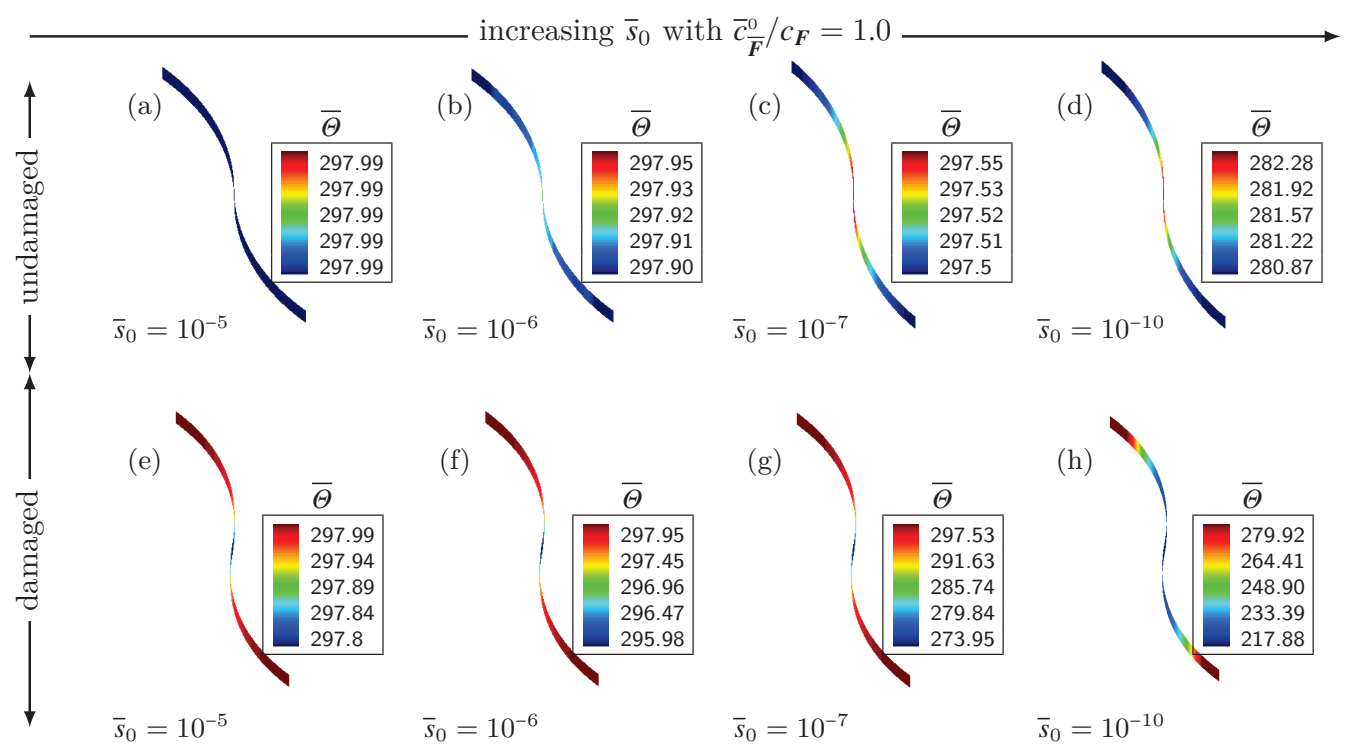

Figure 7: The interface temperature distribution of the strip stretched up to $30 \%$ of its original length for $\bar{\mu} / \mu=\bar{\lambda} / \lambda=2 \mathrm{~mm}, \bar{r}_{Q}^{0}=0.1$ and $c_{\bar{F}}^{0} / c_{\boldsymbol{F}}=1 \mathrm{~mm}$. Results (a)-(d) correspond to the intact interface, whereas results (e)-(h) correspond to the degrading interface.

uniform (see Fig. 5(a)-(d)) than the one along the damaging interface ((see Fig. 5(e)-(h))). Moreover, a degrading interface causes a higher temperature jump across the interface due to the fact that $\bar{r}_{Q}^{0} /\left[1-\bar{D}_{\|}\right]$assumes higher values as damage evolves. One should note that the simplified interface evolution equation of this example takes the form $\overline{\operatorname{Div}}\left(\left[1-\bar{D}_{\|}\right] \overline{\boldsymbol{Q}}\right)=-\llbracket \Theta \rrbracket$, thus a jump in the normal heat flux shall be observed. We point out that, since the interface here is fully dissipative, in contrast to an $\mathrm{HC}$ interface, it allows for a jump in the temperature across the interface. Also, along a non-degrading HC interface with high enough value for the interface conduction coefficient such as $\bar{k}_{0} / k=100 \mathrm{~mm}$, a uniform temperature distribution is achieved. This observation however cannot be made for either an intact or a degrading GI interface. See Fig. $5($ (d) and (h)), respectively.

In the second example the effects of the interface heat capacity are studied. The domain here is stretched up to $30 \%$ of its initial length in 15 equal steps where the total time is $10 \mathrm{~ms}$. Note that for this example $\bar{k}_{0}=\bar{\alpha}=0$, $\bar{c}_{\overline{\boldsymbol{F}}}^{0} / c_{\boldsymbol{F}}=1 \mathrm{~mm}$, and $\bar{s}_{0}$ ranges from $10^{-5}$ to $10^{-10}$. Analogous to the previous example two cases of intact and damaged interface are considered here and shown in Fig. 6 and Fig. 7. From Fig. 6one finds that the intact interface is more capable of maintaining its initial temperature (also see Fig. 7(a)-(d)). On the other hand the domain cools down due to the Gough-Joule effect. These two different responses create the non-uniform temperature distribution in the domain containing the intact interface, as depicted in Fig. 6(a)-(d). On the contrary, a degrading interface can not retain its initial temperature (see Fig. 7( e)-(h)), causing in general a more uniform temperature distribution in the domain as shown in Fig. 6(e)-(h). Note that for this example interface temperature evolution simplifies to 


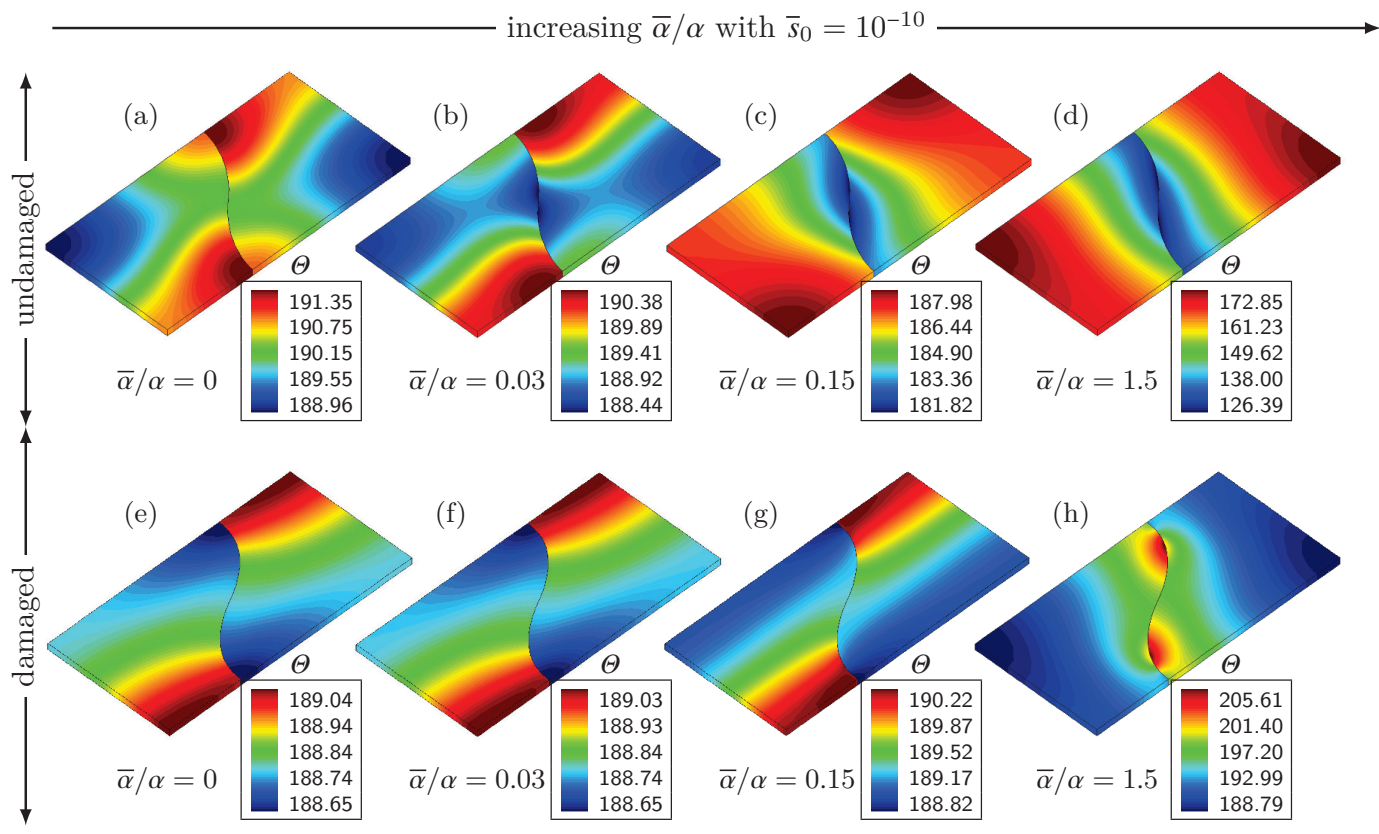

Figure 8: The bulk temperature distribution of the strip stretched up to $100 \%$ of its original length for $\bar{\mu} / \mu=\bar{\lambda} / \lambda=2 \mathrm{~mm}, \bar{r}_{Q}^{0}=0.1$ and $\bar{s}_{0}=10^{-10}$. Results (a)-(d) correspond to the intact interface, whereas results (e)-(h) correspond to the degrading interface.

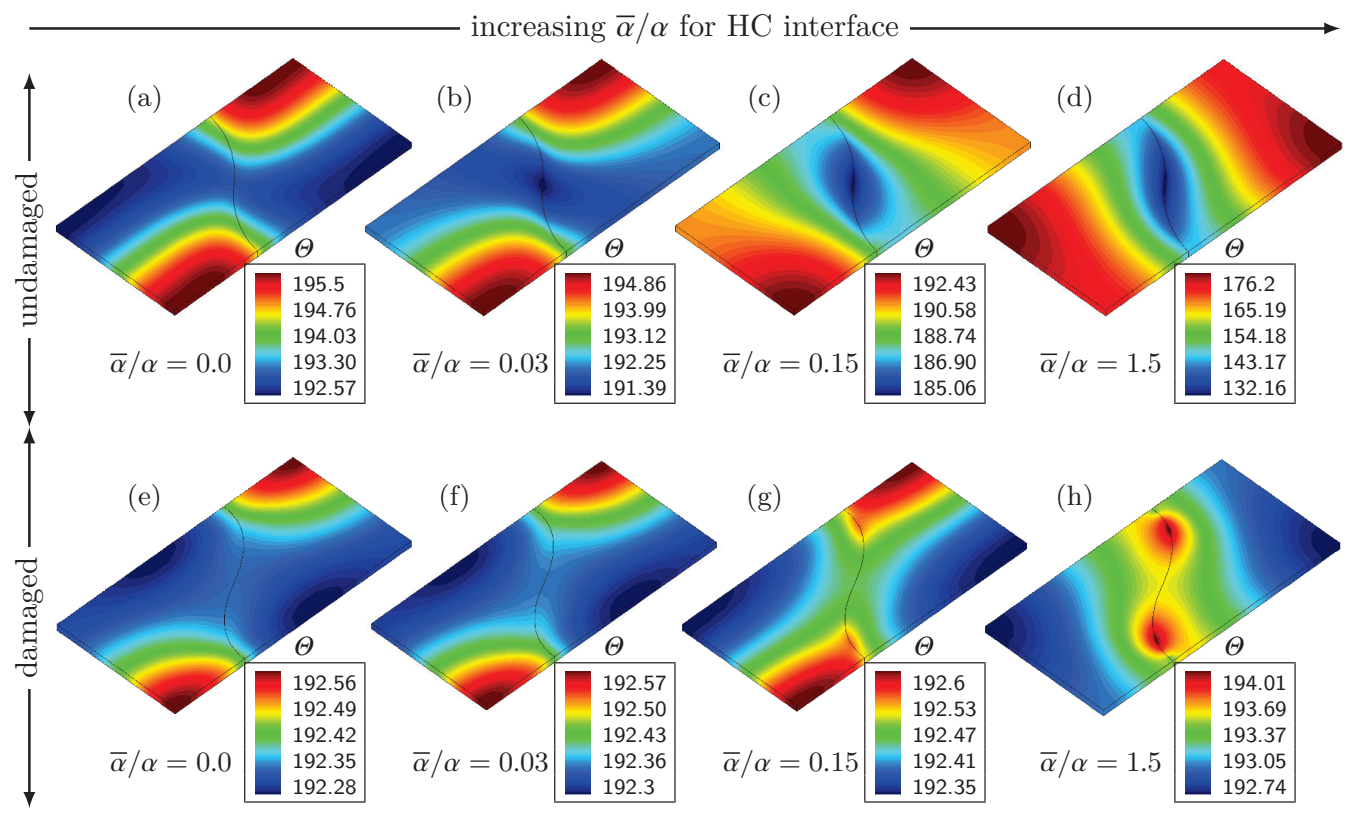

Figure 9: The bulk temperature distribution of the strip stretched up to $100 \%$ of its original length for $\bar{\mu} / \mu=\bar{\lambda} / \lambda=2 \mathrm{~mm}$. Results (a)-(d) correspond to the intact highly-conductive interface, whereas results (e)-(h) correspond to the degrading highly conductive interface. See [21] for further details.

$\left[1-\bar{D}_{\|}\right] \bar{c}_{\overline{\boldsymbol{F}}}^{0} \mathrm{D}_{t} \overline{\boldsymbol{\Theta}}=-\llbracket \boldsymbol{Q} \rrbracket \cdot \overline{\boldsymbol{N}}$, meaning that a jump in the normal heat flux across the interface is present. Here we draw our attention as well to the difference between a thermally GI and $\mathrm{HC}$ interface. As mentioned before an $\mathrm{HC}$ interface 
implies no temperature jump across the interface, and consequently the interface temperature becomes the average of the bulk temperatures on the two sides of the interface. Observing Fig. 6 and Fig. 7 , one concludes that a jump in the temperature is clearly present across the interface, and the interface temperature is not the average of the surrounding bulk temperatures.

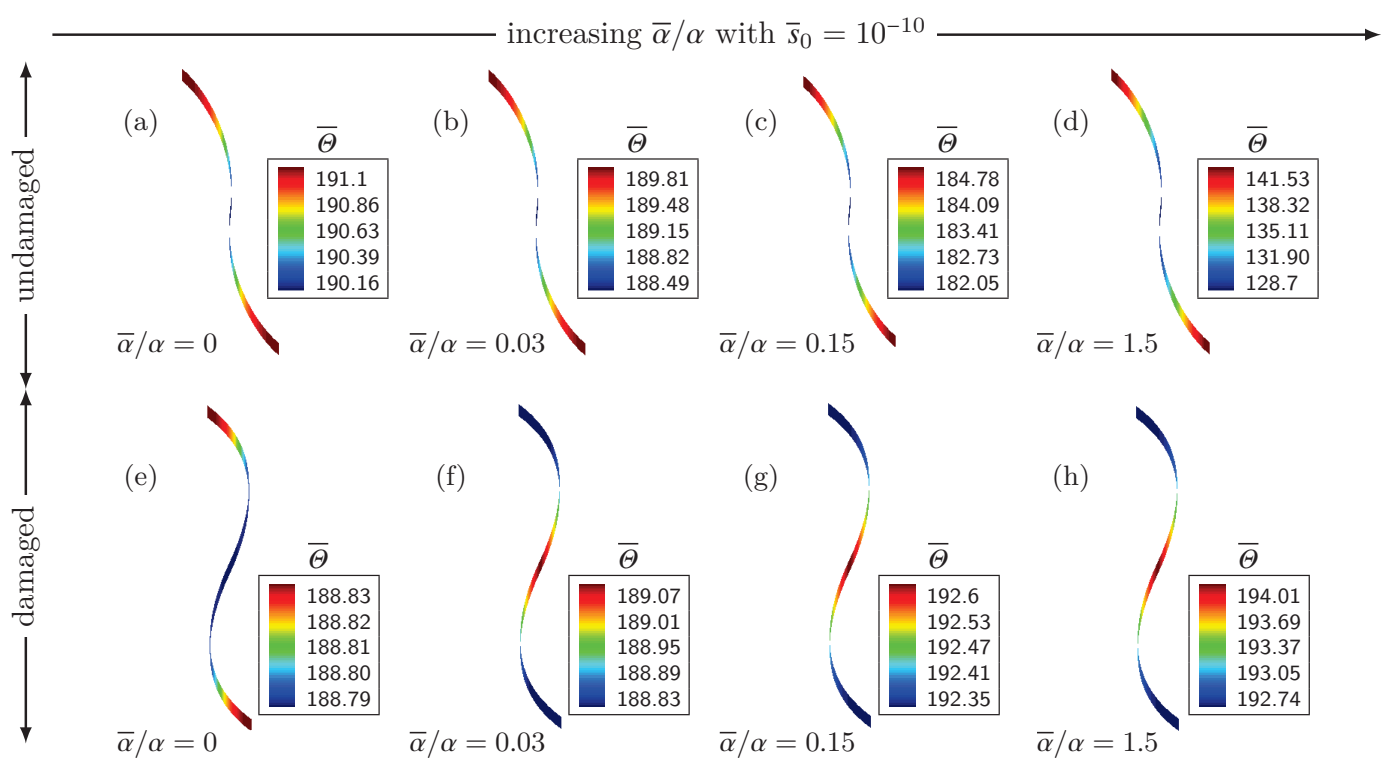

Figure 10: The interface temperature distribution of the strip stretched up to $100 \%$ of its original length for $\bar{\mu} / \mu=\bar{\lambda} / \lambda=2 \mathrm{~mm}, \bar{r}_{Q}^{0}=0.1$ and $\bar{s}_{0}=10^{-10}$. Results (a)-(d) correspond to the intact interface, whereas results (e)-(h) correspond to the degrading interface.

In the final example we turn our attention to the interface Gough-Joule effect by setting $\bar{k}_{0}=\bar{c}_{\bar{F}}^{0}=0, \bar{s}_{0}=10^{-10}$ and ranging $\bar{\alpha} / \alpha$ from 0 to 1.5 . The domain here is stretched up to $100 \%$ of its initial length in 40 equal steps where the total time is $10 \mathrm{~ms}$. The temperature evolution equation in this case simplifies to

$$
\llbracket Q \rrbracket \cdot \overline{\boldsymbol{N}}=\bar{\Theta} \partial_{\bar{\Theta}}\left(\left[1-\bar{D}_{\|}\right] \overline{\boldsymbol{P}}_{0}\right): \mathrm{D}_{t} \overline{\boldsymbol{F}}=\left[\bar{\Theta}\left[1-\bar{D}_{\|}\right] \partial_{\bar{\Theta}} P_{0}-\bar{\Theta} \partial_{\bar{\Theta}} \bar{D}_{\|} \overline{\boldsymbol{P}}_{0}\right]: \mathrm{D}_{t} \overline{\boldsymbol{F}}
$$

From Fig. 8 and Fig. 10 we observe that the intact interface cools down under the increasing load due to the GoughJoule effect more than the degrading interface. Moreover, it is shown that higher temperature jumps and less strong coupling between the interface and bulk temperatures are achieved when the interface is allowed to degrade since $\bar{r}_{Q}^{0} /\left[1-\bar{D}_{\|}\right]$and $\bar{s}_{0} /\left[1-\bar{D}_{\|}\right]$assume higher values as damage evolves (compare Fig. 8 ff)-(h) to Fig. 8 (a)-(d)). Finally, the highest level of interface deformation is observed in this example due to the presence of the thermo-mechanical coupling term $(\bar{\alpha} \neq 0)$ in the interface effective Helmholtz energy. A comparison is also made between a thermally GI and $\mathrm{HC}$ interface by providing Fig. 9 which illustrates a $\mathrm{HC}$ interface under the same conditions as the thermally GI interface shown in Fig. 8 The first observation, as expected, is the vanishing temperature jump across the HC interface. One can also notice that the $\mathrm{HC}$ interface in general retains to a larger extend its initial temperature. Although the 
intact thermally GI and HC interfaces have the lowest temperates in the middle of the interface (see Fig.10.a)-(d) and Fig. 9p, the thermally GI interface temperature, unlike the $\mathrm{HC}$ interface temperature, is not coupled to its surrounding bulk temperatures. This means a thermally GI interface is allowed to be colder or warmer than the bulk (see Fig. 10.h) and Fig. 8 (h) where the interface is colder than the bulk). Note that for a $\mathrm{HC}$ interface the temperature is strongly coupled to that of the bulk by being the average of the adjacent bulk temperatures. Finally the difference between a thermally GI and a HC interface becomes even more pronounced when in-plane degradation is allowed to initiate. Now, a degrading thermally GI interface loses its initial temperature at its two ends more drastically, while on a HC interface the coldest region is the middle of the interface.

\section{Summary and conclusion}

A theoretical and computational framework for continua containing thermally general imperfect and mechanically coherent energetic interfaces was outlined. The corresponding mechanical and thermal weak forms of the balance equations were given. The balance equations were fully discretized using the finite element method in space. The effects of the in-plane degradation on the in- and out-of-plane thermo-mechanical properties of the interface and the overall response of the body were also taken into account by introducing a tangential reduction factor. With the evolution of damage, the in-plane properties, i.e. interface stiffness, heat expansion coefficient, conduction coefficient and heat capacity coefficient are reduced, whereas the out-of-plane properties, i.e. the Kapitza resistance coefficient and sensitivity, are increased. The increase of the out-of-plane thermal properties results in a higher temperature jump and a weaker coupling between interface and bulk temperatures. The tangential damage variable is a function of the equivalent distortion which is non-localized using integral-type averaging.

A series of numerical examples served to elucidate the theory presented in this work. It was shown that the degraded interface undergoes more deformation. In addition, as the heat conduction coefficient is reduced with the damage evolving, higher temperature gradients along the interface were observed. It was also illustrated that an interface retains its initial temperature to a larger extend due to the reduced heat expansion coefficient. The degrading interface was shown to be less capable of being resistant to temperature changes as a result of the reduced specific heat capacity. We also observed that in general, in all the examples higher temperature jumps across the interface and less strong coupling between the interface and bulk temperatures are attributed to damage of the interface. Finally in all the examples, asymptotically quadratic convergence associated with the Newton-Raphson scheme was achieved.

One consequent extension to this work is to study the role of the out-of-plane degradation of the interface material (cohesive damage) on the thermo-mechanical response of a thermally general imperfect and mechanically energetic interface. This includes introducing non-coherent deformation into the current formulation by allowing a displacement 
jump across the interface, which requires the use of a cohesive zone model. Such extension shall be discussed in later contributions.

\section{Acknowledgment}

This research is performed as part of the Energie Campus Nuremberg and supported by funding through the "Bavaria on the Move" initiative of the state of Bavaria. The authors also gratefully acknowledge the support by the Cluster of Excellence "Engineering of Advanced Materials".

\section{Appendix A. Finite element implementation}

In order to apply the finite element method to the present problem, the weak forms Eq. 111 and (12) are discretized in space and time. The time interval $\mathcal{T}$ is subdivided into a set of intervals $\Delta t:=t_{\tau+1}-t_{\tau}$ with

$$
\mathcal{T}=\bigcup_{\tau=0}^{n_{\mathrm{ts}}}\left[t_{\tau}, t_{\tau+1}\right]
$$

where $n_{\mathrm{ts}}$ denotes the number of time steps. The spatial discretization is performed using the Bubnov-Galerkin finite element method. The geometry and temperature of the bulk and interface together with the jump and average of temperature over the interface are approximated as a function of the natural coordinates $\xi \in[-1,1]^{3}$ and $\bar{\xi} \in[-1,1]^{2}$ assigned to the bulk and the interface, respectively. Using standard interpolations according to the isoparametric concepts we obtain

$$
\begin{aligned}
& \left.\boldsymbol{X}\right|_{\mathcal{B}_{0}^{\beta}} \approx \boldsymbol{X}^{\mathrm{h}}(\boldsymbol{\xi})=\sum_{i=1}^{n_{\mathrm{nB}}} N^{i}(\boldsymbol{\xi}) \boldsymbol{X}^{i},\left.\quad \overline{\boldsymbol{X}}\right|_{I_{0}^{\gamma}} \approx \overline{\boldsymbol{X}}^{\mathrm{h}}(\overline{\boldsymbol{\xi}})=\sum_{i=1}^{n_{\mathrm{nL}}} \bar{N}^{i}(\overline{\boldsymbol{\xi}}) \overline{\boldsymbol{X}}^{i}, \\
& \left.\varphi\right|_{\mathcal{B}_{0}^{\beta}} \approx \varphi^{\mathrm{h}}(\xi)=\sum_{i=1}^{n_{\mathrm{nB}}} N^{i}(\xi) \varphi^{i},\left.\quad \bar{\varphi}\right|_{I_{0}^{\gamma}} \approx \bar{\varphi}^{\mathrm{h}}(\bar{\xi})=\sum_{i=1}^{n_{\mathrm{nI}}} \bar{N}^{i}(\bar{\xi}) \bar{\varphi}^{i} \\
& \left.\Theta\right|_{\mathcal{B}_{0}^{\beta}} \approx \Theta^{\mathrm{h}}(\boldsymbol{\xi})=\sum_{i=1}^{n_{\mathrm{nB}}} N^{i}(\boldsymbol{\xi}) \Theta^{i},\left.\quad \bar{\Theta}\right|_{I_{0}^{\gamma}} \approx \bar{\Theta}^{\mathrm{h}}(\bar{\xi})=\sum_{i=1}^{n_{\mathrm{nL}}} \bar{N}^{i}(\bar{\xi}) \bar{\Theta}^{i}, \\
& \left.\llbracket \Theta \rrbracket\right|_{I_{0}^{\gamma}} \approx \llbracket \Theta \rrbracket^{\mathrm{h}}(\bar{\xi})=\sum_{i=1}^{n_{\mathrm{nI}}} \bar{N}^{i}(\bar{\xi}) \llbracket \Theta \rrbracket^{i}, \quad\left\{\left.\Theta \Theta\right|_{I_{0}^{\gamma}} \approx\{\Theta\}^{\mathrm{h}}(\bar{\xi})=\sum_{i=1}^{n_{\mathrm{nI}}} \bar{N}^{i}(\bar{\xi})\{\Theta\}^{i},\right.
\end{aligned}
$$

where $\mathcal{B}_{0}^{\beta}$ and $\mathcal{I}_{0}^{\gamma}$ are the $\beta^{\text {th }}$ and $\gamma^{\text {th }}$ element in the bulk and on the interface, respectively. The shape functions of the bulk and interface elements at a local node $i$ are denoted by $N^{i}$ and $\bar{N}^{i}$, respectively. Every bulk and interface element consists of $n_{\mathrm{nB}}$ and $n_{\mathrm{nI}}$ nodes, respectively.

Now, the fully discrete (spatially and temporally) form of mechanical and thermal residuals associated with the 
global node $I$ are defined by

$$
\begin{aligned}
{\left[{ }^{\mathrm{tot}} \mathbf{R}_{\varphi}^{I}\right]_{\tau+1}=} & \int_{\mathcal{B}_{0}} \boldsymbol{P}_{\tau+1} \cdot \operatorname{Grad} N^{I} \mathrm{~d} V-\int_{\mathcal{B}_{0}} N^{I} \boldsymbol{B}_{\tau+1}^{\mathrm{p}} \mathrm{d} V \\
& +\int_{I_{0}}\left[\left[1-\bar{D}_{\|}\right] \overline{\boldsymbol{P}}_{0}\right]_{\tau+1} \cdot \overline{\operatorname{Grad}} \bar{N}^{I} \mathrm{~d} A-\int_{I_{0}} \bar{N}^{I} \overline{\boldsymbol{B}}_{\tau+1}^{\mathrm{p}} \mathrm{d} A,
\end{aligned}
$$

and

$$
\begin{aligned}
{\left[{ }^{\mathrm{tot}} \mathrm{R}_{\Theta}^{I}\right]_{\tau+1}=} & -\int_{\mathcal{B}_{0}} \boldsymbol{Q}_{\tau+1} \cdot \operatorname{Grad} N^{I}+Q_{\tau+1}^{\mathrm{p}} N^{I} \mathrm{~d} V-\int_{\mathcal{I}_{0}}\left[\left[1-D_{\|}\right] \overline{\boldsymbol{Q}}_{0}\right]_{\tau+1} \cdot \overline{\operatorname{Grad}} \bar{N}^{I}+\bar{Q}_{\tau+1}^{\mathrm{p}} \bar{N}^{I} \mathrm{~d} A \\
& -\int_{\mathcal{B}_{0}} \Theta_{\tau+1}\left[\partial_{\Theta} \boldsymbol{P}\right]_{\tau+1}: \frac{1}{\Delta t}\left[\boldsymbol{F}_{\tau+1}-\boldsymbol{F}_{\tau}\right] N^{I}-c_{\boldsymbol{F}} \frac{1}{\Delta t}\left[\Theta_{\tau+1}-\Theta_{\tau}\right] N^{I} \mathrm{~d} V \\
& -\int_{\mathcal{I}_{0}} \bar{\Theta}_{\tau+1}\left[\partial_{\bar{\Theta}}\left(\left[1-\bar{D}_{\|}\right] \overline{\boldsymbol{P}}_{0}\right)\right]_{\tau+1}: \frac{1}{\Delta t}\left[\overline{\boldsymbol{F}}_{\tau+1}-\overline{\boldsymbol{F}}_{\tau}\right] \bar{N}^{I}-\left[1-\bar{D}_{\|}\right] \bar{c}_{\overline{\boldsymbol{F}}}^{0} \frac{1}{\Delta t}\left[\bar{\Theta}_{\tau+1}-\bar{\Theta}_{\tau}\right] \bar{N}^{I} \mathrm{~d} A \\
& -\int_{\mathcal{I}_{0}}\left[1-\bar{D}_{\|}\right] \frac{1}{\bar{S}_{0}}\left[\bar{\Theta}^{-1}-\left\{\left[\Theta^{-1}\right\}\right] \bar{N}^{I} \mathrm{~d} A\right. \\
& -\int_{\bar{I}_{0}^{-}}\left[1-\bar{D}_{\|}\right] \frac{1}{\bar{r}_{Q}^{0}} \bar{N}^{I} \llbracket \Theta \|_{\tau+1} \mathrm{~d} A+\int_{\mathcal{I}_{0}^{+}}\left[1-\bar{D}_{\|}\right] \frac{1}{\bar{r}_{Q}^{0}} \bar{N}^{I} \llbracket \Theta \rrbracket_{\tau+1} \mathrm{~d} A
\end{aligned}
$$

Note that mechanical and thermal residuals are composed of contributions from both the bulk and interface. Moreover although the integrands of the last two integrals in Eq. A.4 are identical, the domains over which these integrals are taken are different. This is due to the fact that the interface is thermally general imperfect. The global mechanical and thermal residual vectors take the form

$$
{ }^{\text {tot }} \mathbf{R}=\left[\begin{array}{c}
{ }^{\text {tot }} \mathbf{R}_{\varphi} \\
\mathbf{R}_{\Theta} \\
{ }^{\text {tot }} \overline{\mathbf{R}}_{\Theta}
\end{array}\right] \quad \text { with } \quad{ }^{\text {tot }} \mathbf{R}_{\varphi}=\left[\begin{array}{c}
\mathbf{R}_{\varphi}^{1} \\
\vdots \\
\mathbf{R}_{\varphi}^{I} \\
\vdots \\
\mathbf{R}_{\varphi}^{n_{\mathrm{Bn}}}
\end{array}\right]+\left[\begin{array}{c}
\overline{\mathbf{R}}_{\varphi}^{1} \\
\vdots \\
\overline{\mathbf{R}}_{\varphi}^{I} \\
\vdots \\
\overline{\mathbf{R}}_{\varphi}^{n_{\mathrm{In}}}
\end{array}\right] \quad, \quad \mathbf{R}_{\Theta}=\left[\begin{array}{c}
\mathrm{R}_{\Theta}^{1} \\
\vdots \\
\mathrm{R}_{\Theta}^{I} \\
\vdots \\
\mathrm{R}_{\Theta}^{n_{\mathrm{Bn}}}
\end{array}\right] \quad \text { and } \quad{ }^{\text {tot }} \overline{\mathbf{R}}_{\Theta}=\left[\begin{array}{c}
\overline{\mathrm{R}}_{\Theta}^{1} \\
\vdots \\
\overline{\mathrm{R}}_{\Theta}^{I} \\
\vdots \\
\overline{\mathrm{R}}_{\Theta}^{n_{\mathrm{In}}}
\end{array}\right]+\underbrace{\left[\begin{array}{c}
\overline{\mathrm{R}}_{\Theta}^{1^{ \pm}} \\
\vdots \\
\overline{\mathrm{R}}_{\Theta^{ \pm}}^{n_{\mathrm{In}}^{ \pm}}
\end{array}\right]}_{\overline{\mathbf{R}}_{\Theta}}
$$

where $n_{\mathrm{Bn}}$ and $n_{\mathrm{In}}$ denote the number of bulk and interface nodes. The summation operator implies the (conventional) residual assembly of the finite element method. Note that total interface thermal residual ${ }^{\text {tot }} \overline{\mathbf{R}}_{\Theta}$ is composed of contributions from interface residuals corresponding to the degrees of freedom $\bar{\Theta}$ and $\Theta^{ \pm}$, respectively denoted by $\overline{\mathbf{R}}_{\Theta}$ and $\overline{\mathbf{R}}_{\Theta^{ \pm}}$. Both of the above thermal residuals contribute to the total thermal residual, i.e. ${ }^{\text {tot }} \mathbf{R}_{\Theta}=\mathbf{R}_{\Theta}+{ }^{\text {tot }} \overline{\mathbf{R}}_{\Theta}$.

\footnotetext{
${ }^{3}$ In what follows, for the sake of brevity, homogeneous Neumann boundary conditions are assumed and hence, some integrals vanish. The integrals are standard and require no additional care.
} 
The fully-discrete coupled non-linear system of governing equations can be stated as follows:

$$
{ }^{\text {tot }} \mathbf{R}\left({ }^{\text {tot }} \mathbf{d}\right) \stackrel{!}{=} \mathbf{0} \quad \text { with } \quad{ }^{\text {tot }} \mathbf{d}=\left[\begin{array}{c}
\mathbf{d}_{\varphi} \\
\mathbf{d}_{\Theta} \\
\mathbf{d}_{\bar{\Theta}}
\end{array}\right]
$$

where ${ }^{\text {tot }} \mathbf{d}$ is the unknown global vector of spatial coordinates $\mathbf{d}_{\varphi}$ and temperature $\mathbf{d}_{\Theta}$ and $\mathbf{d}_{\bar{\Theta}}$. To solve $A .6,1$, a Newton-Raphson scheme is utilized. Thus, the consistent linearization of the resulting system of equations yields the total (algorithmic) tangent stiffness matrix for every pair of global nodes $I$ and $J$, as

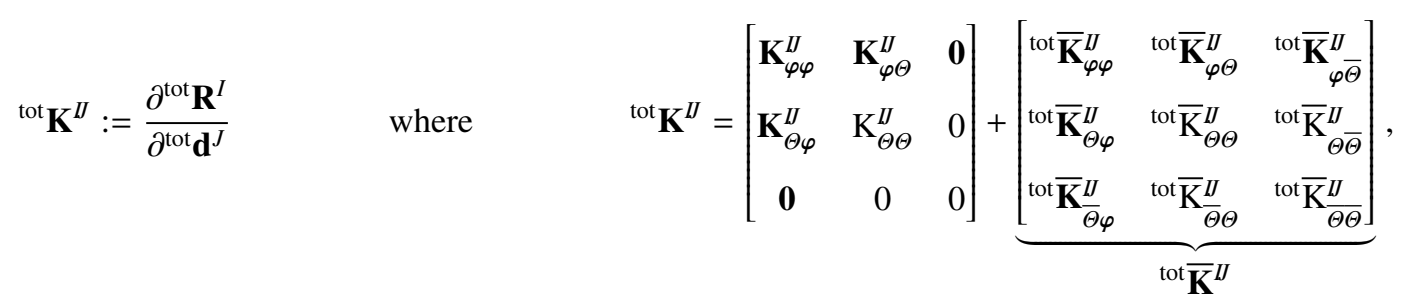

with ${ }^{\text {tot }} \overline{\mathbf{K}}^{I J}$ being defined as

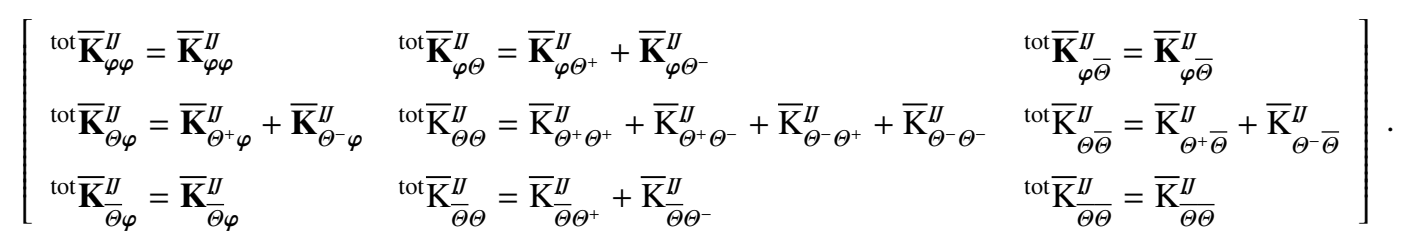

Note that ${ }^{\text {tot }} \mathbf{K}$ is also decomposed into contributions from the bulk and the interface. The bulk contributions to the total stiffness matrix are given as

$$
\begin{aligned}
& \mathbf{K}_{\varphi \varphi}^{I J}=\frac{\partial \mathbf{R}_{\varphi}^{I}}{\partial \varphi^{J}}=\int_{\mathcal{B}_{0}} \operatorname{Grad} N^{I}:\left[\partial_{\boldsymbol{F}} \boldsymbol{P}\right] \cdot \operatorname{Grad} N^{J} \mathrm{~d} V, \\
& \mathbf{K}_{\varphi \Theta}^{I J}=\frac{\partial \mathbf{R}_{\varphi}^{I}}{\partial \Theta^{J}}=\int_{\mathcal{B}_{0}} N^{J}\left[\partial_{\Theta} \boldsymbol{P}\right] \cdot \operatorname{Grad} N^{I} \mathrm{~d} V, \\
& \mathbf{K}_{\Theta \varphi}^{I J}=\frac{\partial \mathbf{R}_{\Theta}^{I}}{\partial \varphi^{J}}=\int_{\mathcal{B}_{0}}-N^{I}\left[\frac{\partial\left(\Theta \partial_{\Theta} \boldsymbol{P}: \mathrm{D}_{t} \boldsymbol{F}\right)}{\partial \boldsymbol{F}}\right] \cdot \operatorname{Grad} N^{J}-\operatorname{Grad} N^{I} \cdot\left[\partial_{\boldsymbol{F}} \boldsymbol{Q}\right] \cdot \operatorname{Grad} N^{J} \mathrm{~d} V, \\
& \mathrm{~K}_{\Theta \Theta}^{I J}=\frac{\partial \mathrm{R}_{\Theta}^{I}}{\partial \Theta^{J}}=\int_{\mathcal{B}_{0}}-\operatorname{Grad} N^{I} \cdot\left[\frac{\partial \boldsymbol{Q}}{\partial \operatorname{Grad} \Theta}\right] \cdot \operatorname{Grad} N^{J}+N^{I}\left[\frac{c_{\boldsymbol{F}}}{\Delta t}-\left[\frac{\partial\left(\Theta \partial_{\Theta} \boldsymbol{P}: \mathrm{D}_{t} \boldsymbol{F}\right)}{\partial \Theta}\right]\right] N^{J} \mathrm{~d} V .
\end{aligned}
$$

The interface contributions to the total stiffness matrix consist of local ${ }^{10} \overline{\mathbf{K}}$ and non-local ${ }^{\text {nloc }} \overline{\mathbf{K}}$ contributions. The 
local contributions ${ }^{10} \overline{\mathbf{K}}$, uniquely derived for a GI interface are as follows:

$$
\begin{aligned}
& { }^{l{ }^{l o c}} \overline{\mathrm{K}}_{\Theta^{ \pm} \bar{\Theta}}^{I J}=\frac{\partial \overline{\mathrm{R}}_{\Theta}^{I^{ \pm}}}{\partial \bar{\Theta}^{J}}=\int_{I_{0}^{ \pm}}\left[1-\bar{D}_{\|}\left(\overline{\boldsymbol{x}}_{\mathrm{r}}\right)\right] \frac{1}{2 \bar{s}_{0}} \frac{1}{\bar{\Theta}^{2}} \bar{N}^{I} \bar{N}^{J} \mathrm{~d} A_{\mathrm{r}}, \\
& { }^{10 c} \overline{\mathrm{K}}_{\bar{\Theta} \Theta^{ \pm}}^{I J}=\frac{\partial \overline{\mathrm{R}}_{\bar{\Theta}}^{I}}{\partial \Theta^{J^{ \pm}}}=\int_{\mathcal{I}_{0}}\left[1-\bar{D}_{\|}\left(\overline{\boldsymbol{x}}_{\mathrm{r}}\right)\right] \frac{1}{2 \bar{s}_{0}} \frac{1}{\left[\Theta^{ \pm}\right]^{2}} \bar{N}^{I} \bar{N}^{J} \mathrm{~d} A_{\mathrm{r}}, \\
& { }^{\mathrm{loc}} \overline{\mathrm{K}}_{\Theta^{ \pm} \Theta^{ \pm}}^{I J}=\frac{\partial \overline{\mathrm{R}}_{\Theta}^{I^{ \pm}}}{\partial \Theta^{J^{ \pm}}}=\int_{I_{0}^{ \pm}}\left[1-\bar{D}_{\|}\left(\overline{\boldsymbol{x}}_{\mathrm{r}}\right)\right] \frac{1}{4 \bar{s}_{0}} \frac{1}{\left[\Theta^{ \pm \pm}\right]^{2}} \bar{N}^{I} \bar{N}^{J} \mathrm{~d} A_{\mathrm{r}} \pm \pm \int_{I_{0}^{ \pm}}\left[1-\bar{D}_{\|}\left(\overline{\boldsymbol{x}}_{\mathrm{r}}\right)\right] \frac{1}{\bar{r}_{Q}^{0}} \bar{N}^{I} \bar{N}^{J} \mathrm{~d} A_{\mathrm{r}},
\end{aligned}
$$

where $\mathrm{d} A_{\mathrm{r}}=\mathrm{d} A\left(\overline{\boldsymbol{x}}_{\mathrm{r}}\right)$. The rest of interface local contributions to the total stiffness matrix are given as

$$
\begin{aligned}
& { }^{{ }^{\circ o}} \overline{\mathbf{K}}_{\varphi \varphi}^{I J}=\frac{\partial \overline{\mathbf{R}}_{\varphi}^{I}}{\partial \bar{\varphi}^{J}}=\int_{\mathcal{I}_{0}} \overline{\operatorname{Grad}} \bar{N}^{I} \cdot\left[1-\bar{D}_{\|}\left(\overline{\boldsymbol{x}}_{\mathrm{r}}\right)\right] \partial_{\overline{\boldsymbol{F}}} \overline{\boldsymbol{P}}_{0}\left(\overline{\boldsymbol{x}}_{\mathrm{r}}\right) \cdot \overline{\operatorname{Grad}} \bar{N}^{J} \mathrm{~d} A_{\mathrm{r}}, \\
& { }^{100} \overline{\mathbf{K}}_{\varphi \bar{\Theta}}^{I J}=\frac{\partial \overline{\mathbf{R}}_{\varphi}^{I}}{\partial \bar{\Theta}^{J}}=\int_{I_{0}} \bar{N}^{J}\left[1-\bar{D}_{\|}\left(\overline{\boldsymbol{x}}_{\mathrm{r}}\right)\right] \partial_{\bar{\Theta}} \overline{\boldsymbol{P}}_{0}\left(\overline{\boldsymbol{x}}_{\mathrm{r}}\right) \cdot \overline{\operatorname{Grad}} \bar{N}^{I} \mathrm{~d} A_{\mathrm{r}}, \\
& { }^{10} \overline{\mathbf{K}}_{\bar{\Theta} \varphi}^{I J}=\frac{\partial \overline{\mathrm{R}}_{\Theta}^{I}}{\partial \overline{\boldsymbol{\varphi}}^{J}}=\int_{\mathcal{I}_{0}}-\bar{N}^{I}\left[1-\bar{D}_{\|}\left(\overline{\boldsymbol{x}}_{\mathrm{r}}\right)\right]\left[\frac{\partial\left(\bar{\Theta} \partial_{\bar{\Theta}} \overline{\boldsymbol{P}}_{0}\left(\overline{\boldsymbol{x}}_{\mathrm{r}}\right): \mathrm{D}_{t} \overline{\boldsymbol{F}}\right)}{\partial \overline{\boldsymbol{F}}}\right] \cdot \overline{\operatorname{Grad}} \bar{N}^{J} \mathrm{~d} A_{\mathrm{r}} \\
& -\int_{\mathcal{I}_{0}} \bar{N}^{I}\left[-\partial_{\bar{\Theta}} \overline{\mathscr{D}}_{\|}\left(\overline{\boldsymbol{x}}_{\mathrm{r}}\right)\right]\left[\frac{\partial\left(\bar{\Theta} \overline{\boldsymbol{P}}_{0}\left(\overline{\boldsymbol{x}}_{\mathrm{r}}\right): \mathrm{D}_{t} \overline{\boldsymbol{F}}\right)}{\partial \overline{\boldsymbol{F}}}\right] \cdot \overline{\mathrm{Grad}} \bar{N}^{J} \mathrm{~d} A_{\mathrm{r}} \\
& -\int_{I_{0}} \overline{\operatorname{Grad}} \bar{N}^{I} \cdot\left[1-\bar{D}_{\|}\left(\overline{\boldsymbol{x}}_{\mathrm{r}}\right)\right]\left[\partial_{\overline{\boldsymbol{F}}} \overline{\boldsymbol{Q}}_{0}\left(\overline{\boldsymbol{x}}_{\mathrm{r}}\right)\right] \cdot \overline{\operatorname{Grad}} \bar{N}^{J} \mathrm{~d} A_{\mathrm{r}}, \\
& { }^{\mathrm{loc}} \overline{\mathrm{K}}_{\overline{\Theta \Theta}}^{I J}=\frac{\partial \overline{\mathrm{R}}_{\Theta}^{I}}{\partial \bar{\Theta}^{J}}=\int_{I_{0}}-\overline{\operatorname{Grad}} \bar{N}^{I} \cdot\left[1-\bar{D}_{\|}\left(\overline{\boldsymbol{x}}_{\mathrm{r}}\right)\right]\left[\frac{\partial \overline{\boldsymbol{Q}}_{0}\left(\overline{\boldsymbol{x}}_{\mathrm{r}}\right)}{\partial \overline{\operatorname{Grad} \Theta}}\right] \cdot \overline{\operatorname{Grad}} \bar{N}^{J} \mathrm{~d} A_{\mathrm{r}} \\
& +\int_{\mathcal{I}_{0}} \bar{N}^{I}\left[1-\bar{D}_{\|}\left(\overline{\boldsymbol{x}}_{\mathrm{r}}\right)\right]\left[\frac{\bar{c}_{\overline{\boldsymbol{F}}}^{0}}{\Delta t}+\frac{1}{\bar{s}_{0} \bar{\Theta}^{2}}-\left[\frac{\partial\left(\bar{\Theta} \partial_{\bar{\Theta}} \overline{\boldsymbol{P}}_{0}\left(\overline{\boldsymbol{x}}_{\mathrm{r}}\right): \mathrm{D}_{t} \overline{\boldsymbol{F}}\right)}{\partial \bar{\Theta}}\right]\right] \bar{N}^{J} \mathrm{~d} A_{\mathrm{r}} \\
& -\int_{\mathcal{I}_{0}} \bar{N}^{I}\left[-\partial_{\bar{\Theta}} \overline{\mathscr{D}}_{\|}\left(\overline{\boldsymbol{x}}_{\mathrm{r}}\right)\right]\left[\frac{\partial\left(\bar{\Theta} \overline{\boldsymbol{P}}_{0}\left(\overline{\boldsymbol{x}}_{\mathrm{r}}\right): \mathrm{D}_{t} \overline{\boldsymbol{F}}\right)}{\partial \bar{\Theta}}\right] \bar{N}^{J} \mathrm{~d} A_{\mathrm{r}},
\end{aligned}
$$

where $\partial_{\bar{\Theta}} \overline{\mathscr{D}}_{\|}$, using Eq. $\sqrt{6}_{2,3}$, is computed as follows:

$$
\partial_{\bar{\Theta}} \overline{\mathscr{D}}_{\|}\left(\overline{\boldsymbol{x}}_{\mathrm{r}}\right)=\overline{\mathscr{D}}_{\|}^{\prime} \partial_{\bar{\Theta}} F_{\text {nloc }} \Longrightarrow \partial_{\bar{\Theta}} F_{\text {nloc }}\left(\overline{\boldsymbol{x}}_{\mathrm{r}}\right)=\int_{\mathcal{I}_{0}} \omega\left(\overline{\boldsymbol{x}}_{\mathrm{r}}, \overline{\boldsymbol{x}}_{\mathrm{s}}\right) \frac{1}{\bar{E} F_{\text {loc }}\left(\overline{\boldsymbol{x}}_{\mathrm{s}}\right)} \partial_{\bar{\Theta}} \bar{\Psi}^{0}\left(\overline{\boldsymbol{x}}_{\mathrm{s}}\right) \mathrm{d} A_{\mathrm{s}} \text { with } \overline{\mathscr{D}}_{\|}^{\prime}=\partial_{F_{\text {nloc }}} \overline{\mathscr{D}}_{\|} .
$$


Next, the non-local corrections to the interface stiffness matrix are given as

$$
\begin{aligned}
& { }^{\text {nloc }} \overline{\mathbf{K}}_{\varphi \varphi}^{I J}=\frac{\partial \overline{\mathbf{R}}_{\varphi}^{I}}{\partial \bar{\varphi}^{J}}=\int_{I_{0}} \overline{\operatorname{Grad}} \bar{N}^{I} \cdot \overline{\boldsymbol{P}}_{0}\left(\overline{\boldsymbol{x}}_{\mathrm{r}}\right) \otimes\left[-\partial_{\varphi^{J}} \overline{\mathscr{D}}_{\|}\left(\overline{\boldsymbol{x}}_{\mathrm{r}}\right)\right] \mathrm{d} A_{\mathrm{r}}, \\
& { }^{\mathrm{nloc}} \overline{\mathbf{K}}_{\varphi \bar{\Theta}}^{I J}=\frac{\partial \overline{\mathbf{R}}_{\varphi}^{I}}{\partial \bar{\Theta}^{J}}=\int_{\mathcal{I}_{0}}\left[-\partial_{\bar{\Theta}^{J}} \overline{\mathscr{D}}_{\|}\left(\overline{\boldsymbol{x}}_{\mathrm{r}}\right)\right] \overline{\boldsymbol{P}}_{0}\left(\overline{\boldsymbol{x}}_{\mathrm{r}}\right) \cdot \overline{\operatorname{Grad}} \bar{N}^{I} \mathrm{~d} A_{\mathrm{r}}, \\
& { }^{\text {nloc }} \overline{\mathbf{K}}_{\bar{\Theta} \varphi}^{I J}=\frac{\partial \overline{\mathrm{R}}_{\Theta}^{I}}{\partial \overline{\boldsymbol{\varphi}}^{J}}=\int_{\mathcal{I}_{0}}-\bar{N}^{I} \bar{\Theta} \mathrm{D}_{t} \overline{\boldsymbol{F}}:\left[-\partial_{\bar{\Theta}} \overline{\boldsymbol{P}}_{0} \otimes \partial_{\bar{\varphi}^{J}} \overline{\mathscr{D}}_{\|}-\overline{\boldsymbol{P}}_{0} \otimes \partial_{\bar{\varphi}^{J}} \overline{\mathscr{D}}_{\|}\right]_{\overline{\boldsymbol{x}}_{\mathrm{r}}} \mathrm{d} A_{\mathrm{r}} \\
& -\int_{I_{0}} \overline{\operatorname{Grad}} \bar{N}^{I} \cdot \overline{\boldsymbol{Q}}_{0}\left(\overline{\boldsymbol{x}}_{\mathrm{r}}\right) \otimes\left[-\partial_{\bar{\varphi}^{J}} \overline{\mathscr{D}}_{\|}\left(\overline{\boldsymbol{x}}_{\mathrm{r}}\right)\right]+\bar{N}^{I}\left[-\partial_{\bar{\varphi}^{J}} \overline{\mathscr{D}}_{\|}\left(\overline{\boldsymbol{x}}_{\mathrm{r}}\right)\right]\left[\bar{c}_{\overline{\boldsymbol{F}}}^{0} \mathrm{D}_{t} \bar{\Theta}-\frac{1}{\bar{s}_{0}}\left[\bar{\Theta}^{-1}-\{\Theta\}^{-1}\right]\right]_{\bar{x}_{\mathrm{r}}} \mathrm{d} A_{\mathrm{r}}, \\
& { }^{\mathrm{nloc}} \overline{\mathbf{K}}_{\Theta^{ \pm} \varphi}^{I J}=\frac{\partial \overline{\mathrm{R}}_{\Theta}^{I^{ \pm}}}{\partial \bar{\varphi}^{J}}=\int_{I_{0}^{ \pm}} \bar{N}^{I}\left[-\partial_{\bar{\varphi}^{J}} \overline{\mathscr{D}}_{\|}\left(\overline{\boldsymbol{x}}_{\mathrm{r}}\right)\right]\left[\frac{1}{2 \overline{\boldsymbol{s}}_{0}}\left[\bar{\Theta}^{-1}-\{\Theta\}^{-1}\right] \pm \frac{1}{\bar{r}_{Q}^{0}} \llbracket \Theta \rrbracket\right]_{\overline{\boldsymbol{x}}_{\mathrm{r}}} \mathrm{d} A_{\mathrm{r}}, \\
& { }^{\mathrm{nloc}} \overline{\mathrm{K}}_{\overline{\Theta \Theta}}^{I J}=\frac{\partial \overline{\mathrm{R}}_{\Theta}^{I}}{\partial \bar{\Theta}^{J}}=\int_{I_{0}}-\overline{\mathrm{Grad}} \bar{N}^{I} \cdot\left[-\partial_{\bar{\Theta}^{J}} \overline{\mathscr{D}}_{\|}\left(\overline{\boldsymbol{x}}_{\mathrm{r}}\right)\right] \overline{\boldsymbol{Q}}_{0}\left(\overline{\boldsymbol{x}}_{\mathrm{r}}\right)-\bar{N}^{I} \bar{\Theta}\left[-\partial_{\bar{\Theta}^{J}} \overline{\mathscr{D}}_{\|} \partial_{\bar{\Theta}} \overline{\boldsymbol{P}}_{0}-\partial_{\bar{\Theta}^{J}} \overline{\mathscr{D}}_{\|} \overline{\boldsymbol{P}}_{0}\right]_{\overline{\boldsymbol{x}}_{\mathrm{r}}}: \mathrm{D}_{t} \overline{\boldsymbol{F}}\left(\overline{\boldsymbol{x}}_{\mathrm{r}}\right) \mathrm{d} A_{\mathrm{r}} \\
& +\int_{\mathcal{I}_{0}} \bar{N}^{I}\left[-\partial_{\bar{\Theta}^{J}} \overline{\mathscr{D}}_{\|}\left(\overline{\boldsymbol{x}}_{\mathrm{r}}\right)\right]\left[\bar{c}_{\overline{\boldsymbol{F}}}^{0} \mathrm{D}_{t} \bar{\Theta}-\frac{1}{\bar{s}_{0}}\left[\bar{\Theta}^{-1}-\left\{\Theta^{-1}\right\}\right]\right]_{\bar{x}_{\mathrm{r}}} \mathrm{d} A_{\mathrm{r}},
\end{aligned}
$$

where $\mathrm{D}_{t} \overline{\boldsymbol{F}}=\left[\overline{\boldsymbol{F}}_{\tau+1}-\overline{\boldsymbol{F}}_{\tau}\right] / \Delta t$ and $\mathrm{D}_{t} \bar{\Theta}=\left[\bar{\Theta}_{\tau+1}-\bar{\Theta}_{\tau}\right] / \Delta t$. The notation $[\{\bullet\}]_{\bar{x}_{\mathrm{r}}}$ means that the quantity $\{\bullet\}$ is evaluated at point $\overline{\boldsymbol{x}}_{\mathrm{r}}$. In the derivation of the non-local corrections to the interface stiffness matrix the first and second derivatives of damage variable with respect to the nodal coordinates and temperature $\bar{\varphi}^{J}$ and $\bar{\Theta}^{J}$, at an arbitrary point $\bar{x}_{\mathrm{r}}$, on the interface, using Eq. (6) 2,3, are calculated as follows:

$$
\begin{aligned}
& \partial_{\bar{\Theta}^{J}} \overline{\mathscr{D}}_{\|}\left(\overline{\boldsymbol{x}}_{\mathrm{r}}\right)=\overline{\mathscr{D}}_{\|}^{\prime} \partial_{\bar{\Theta}^{J}} F_{\text {nloc }} \Longrightarrow \partial_{\bar{\Theta}^{J}} F_{\text {nloc }}\left(\overline{\boldsymbol{x}}_{\mathrm{r}}\right)=\int_{\mathcal{I}_{0}} \omega\left(\overline{\boldsymbol{x}}_{\mathrm{r}}, \overline{\boldsymbol{x}}_{\mathrm{s}}\right) \frac{1}{\bar{E} F_{\text {loc }}\left(\overline{\boldsymbol{x}}_{\mathrm{s}}\right)} \partial_{\bar{\Theta}} \bar{\Psi}^{0}\left(\overline{\boldsymbol{x}}_{\mathrm{s}}\right) \bar{N}^{J}\left(\overline{\boldsymbol{x}}_{\mathrm{s}}\right) \mathrm{d} A_{\mathrm{s}} \\
& \partial_{\bar{\varphi}^{J}} \overline{\mathscr{D}}_{\|}\left(\overline{\boldsymbol{x}}_{\mathrm{r}}\right)=\overline{\mathscr{D}}_{\|}^{\prime} \partial_{\bar{\varphi}^{J}} F_{\text {nloc }} \Longrightarrow \partial_{\bar{\varphi}^{J}} F_{\text {nloc }}\left(\overline{\boldsymbol{x}}_{\mathrm{r}}\right)=\int_{\mathcal{I}_{0}} \omega\left(\overline{\boldsymbol{x}}_{\mathrm{r}}, \overline{\boldsymbol{x}}_{\mathrm{s}}\right) \frac{1}{\bar{E} F_{\mathrm{loc}}\left(\overline{\boldsymbol{x}}_{\mathrm{s}}\right)} \partial_{\overline{\boldsymbol{F}}} \bar{\Psi}^{0}\left(\overline{\boldsymbol{x}}_{\mathrm{s}}\right) \cdot \overline{\operatorname{Grad}} \bar{N}^{J}\left(\overline{\boldsymbol{x}}_{\mathrm{s}}\right) \mathrm{d} A_{\mathrm{s}},
\end{aligned}
$$

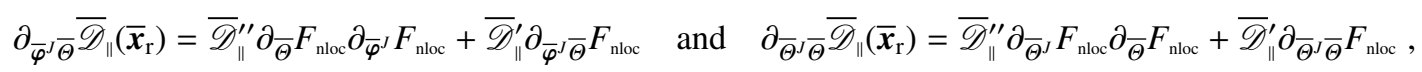

where $\overline{\mathscr{D}}_{\|}^{\prime \prime}=\partial_{F_{\text {nloc }}} \overline{\mathscr{D}}_{\|}^{\prime}, \mathrm{d} A_{\mathrm{s}}=\mathrm{d} A\left(\overline{\boldsymbol{x}}_{\mathrm{s}}\right)$, and

$$
\begin{aligned}
& \partial_{\bar{\varphi}^{J} \bar{\Theta}} F_{\text {nloc }}\left(\overline{\boldsymbol{x}}_{\mathrm{r}}\right)=\int_{\mathcal{I}_{0}} \omega\left(\overline{\boldsymbol{x}}_{\mathrm{r}}, \overline{\boldsymbol{x}}_{\mathrm{s}}\right)\left[\frac{-1}{\bar{E}^{2} F_{\mathrm{loc}}^{3}} \partial_{\overline{\boldsymbol{F}}} \bar{\Psi}^{0} \partial_{\bar{\Theta}} \bar{\Psi}^{0}+\frac{1}{\bar{E} F_{\mathrm{loc}}} \partial_{\overline{\boldsymbol{F}}} \bar{\Theta}^{0} \bar{\Psi}_{\overline{\boldsymbol{x}}_{\mathrm{s}}} \cdot \overline{\operatorname{Grad}} \bar{N}^{J}\left(\overline{\boldsymbol{x}}_{\mathrm{s}}\right) \mathrm{d} A_{\mathrm{s}},\right. \\
& \partial_{\bar{\Theta}^{J} \bar{\Theta}} F_{\mathrm{nloc}}\left(\overline{\boldsymbol{x}}_{\mathrm{r}}\right)=\int_{\mathcal{I}_{0}} \omega\left(\overline{\boldsymbol{x}}_{\mathrm{r}}, \overline{\boldsymbol{x}}_{\mathrm{s}}\right)\left[\frac{-1}{\bar{E}^{2} F_{\mathrm{loc}}^{3}}\left[\partial_{\bar{\Theta}} \bar{\Psi}^{0}\right]^{2}+\frac{1}{\bar{E} F_{\mathrm{loc}}} \partial_{\bar{\Theta}} \bar{\Theta}^{0} \bar{\Psi}_{\overline{\boldsymbol{x}}_{\mathrm{s}}} \bar{N}^{J}\left(\overline{\boldsymbol{x}}_{\mathrm{s}}\right) \mathrm{d} A_{\mathrm{s}} .\right.
\end{aligned}
$$

Similarly the notation $[\{\bullet\}]_{\overline{\boldsymbol{x}}_{\mathrm{s}}}$ means that the quantity $\{\bullet\}$ is evaluated at point $\overline{\boldsymbol{x}}_{\mathrm{s}}$. Note that by using the derivatives A.14 - A.18 in the non-local corrections A.13, the double integrals are introduced into the formulation due to non-locality of the damage model. Furthermore during unloading we set $\overline{\mathscr{D}}^{\prime}=\overline{\mathscr{D}}^{\prime \prime}=0$. 


\section{Appendix B. Constitutive relations in the bulk and on the interface}

\section{bulk}

$\Psi=\frac{1}{2} \lambda \ln ^{2} J+\frac{1}{2} \mu[\boldsymbol{F}: \boldsymbol{F}-3-2 \ln J]$

$-3 \alpha \kappa\left[\Theta-\Theta_{0}\right] J^{-1} \ln J+c_{F}\left[\Theta-\Theta_{0}-\Theta \ln \left(\Theta / \Theta_{0}\right)\right]-\Xi_{0}\left[\Theta-\Theta_{0}\right]$

$\boldsymbol{P}=\lambda \ln J \boldsymbol{F}^{-\mathrm{t}}+\mu\left[\boldsymbol{F}-\boldsymbol{F}^{-\mathrm{t}}\right]-3 \alpha \kappa J^{-1}\left[\Theta-\Theta_{0}\right][1-\ln J] \boldsymbol{F}^{-\mathrm{t}}$

$\Xi=3 \alpha \kappa J^{-1} \ln J+c_{F} \ln \left(\Theta / \Theta_{0}\right)+\Xi_{0}$

$\partial_{\boldsymbol{F}} \boldsymbol{P}=\lambda\left[\boldsymbol{F}^{-\mathrm{t}} \otimes \boldsymbol{F}^{-\mathrm{t}}+\ln J \mathbb{D}\right]+\mu[\mathbb{I}-\mathbb{D}]+3 \alpha \kappa\left[\Theta-\Theta_{0}\right]\left[J^{-1}[2-\ln J] \boldsymbol{F}^{-\mathrm{t}} \otimes \boldsymbol{F}^{-\mathrm{t}}-J^{-1}[1-\ln J] \mathbb{D}\right]$

$\partial_{\Theta} \boldsymbol{P}=-3 \alpha \kappa J^{-1}[1-\ln J] \boldsymbol{F}^{-\mathrm{t}}$

$\partial_{\Theta}\left(\Theta \partial_{\Theta} \boldsymbol{P}: \mathrm{D}_{t} \boldsymbol{F}\right)=-3 \alpha \kappa J^{-1}[1-\ln J] \operatorname{div} \boldsymbol{V}$

$\partial_{\boldsymbol{F}}\left(\Theta \partial_{\Theta} \boldsymbol{P}: \mathrm{D}_{t} \boldsymbol{F}\right)=-3 \alpha \kappa \Theta\left[J^{-1}[\ln J-2] \operatorname{div} \boldsymbol{V} \boldsymbol{F}^{-\mathrm{t}}+J^{-1}[1-\ln J]\left[\mathrm{D}_{t} \boldsymbol{F}: \mathbb{D}+[1 / \Delta t] \boldsymbol{F}^{-\mathrm{t}}: \mathbb{I}\right]\right]$

$\left.\partial_{\boldsymbol{F}} \boldsymbol{Q}=-J k\left[\boldsymbol{F}^{-1} \cdot \boldsymbol{F}^{-\mathrm{t}} \otimes \boldsymbol{F}^{-\mathrm{t}}+\mathbb{B}\right]\right]_{-} \operatorname{Grad} \Theta \quad, \quad \partial_{\mathrm{Grad} \Theta} \boldsymbol{Q}=-J k \boldsymbol{G}$

$\mathbb{D}=\partial_{\boldsymbol{F}} \boldsymbol{F}^{-\mathrm{t}}=-\boldsymbol{F}^{-\mathrm{t}} \otimes \boldsymbol{F}^{-1}$

,$\quad \mathbb{I}=\partial_{F} F=i \bar{\otimes} I$

$\mathbb{B}=\partial_{\boldsymbol{F}}\left(\boldsymbol{F}^{-1} \cdot \boldsymbol{F}^{-\mathrm{t}}\right)=-\left[\boldsymbol{F}^{-1} \bar{\otimes} \boldsymbol{F}^{-1}\right] \cdot \boldsymbol{F}^{-\mathrm{t}}-\boldsymbol{F}^{-1} \cdot\left[\boldsymbol{F}^{-\mathrm{t}} \underline{\otimes} \boldsymbol{F}^{-\mathrm{t}}\right] \quad, \quad \boldsymbol{G}=\boldsymbol{F}^{-1} \cdot \boldsymbol{F}^{-\mathrm{t}}$

interface

$\bar{\Psi}^{0}=\frac{1}{2} \bar{\lambda} \ln ^{2} \bar{J}+\frac{1}{2} \bar{\mu}[\overline{\boldsymbol{F}}: \overline{\boldsymbol{F}}-2-2 \ln \bar{J}]$

$-2 \bar{\alpha} \bar{\kappa}\left[\bar{\Theta}-\bar{\Theta}_{0}\right] \bar{J}^{-1} \ln \bar{J}+\bar{c}_{\bar{F}}^{0}\left[\bar{\Theta}-\bar{\Theta}_{0}-\bar{\Theta} \ln \left(\bar{\Theta} / \bar{\Theta}_{0}\right)\right]-\bar{\Xi}_{\mathrm{s}}\left[\bar{\Theta}-\bar{\Theta}_{0}\right]$

$\overline{\boldsymbol{P}}_{0}=\partial_{\overline{\boldsymbol{F}}} \bar{\Psi}^{0}=\bar{\lambda} \ln \bar{J} \overline{\boldsymbol{F}}^{-\mathrm{t}}+\bar{\mu}\left[\overline{\boldsymbol{F}}-\overline{\boldsymbol{F}}^{-\mathrm{t}}\right]-2 \bar{\alpha} \overline{\boldsymbol{K}}^{-1}\left[\bar{\Theta}-\bar{\Theta}_{0}\right][1-\ln \bar{J}] \overline{\boldsymbol{F}}^{-\mathrm{t}}$

$\bar{\Xi}_{0}=2 \bar{\alpha} \bar{\kappa} \bar{J}^{-1} \ln \bar{J}+\bar{c}_{\bar{F}}^{0} \ln \left(\bar{\Theta} / \bar{\Theta}_{0}\right)+\bar{\Xi}_{\mathrm{s}}$

$\partial_{\bar{\Theta}} \bar{\Psi}^{0}=-\bar{\Xi}_{0}$

$\partial_{\overline{\boldsymbol{F}}} \overline{\boldsymbol{P}}_{0}=\bar{\lambda}\left[\overline{\boldsymbol{F}}^{-\mathrm{t}} \otimes \overline{\boldsymbol{F}}^{-\mathrm{t}}+\ln \bar{J} \overline{\mathbb{D}}\right]+\bar{\mu}[\overline{\mathbb{I}}-\overline{\mathbb{D}}]+2 \bar{\alpha} \bar{\kappa}\left[\bar{\Theta}-\bar{\Theta}_{0}\right]\left[\bar{J}^{-1}[2-\ln \bar{J}] \overline{\boldsymbol{F}}^{-\mathrm{t}} \otimes \overline{\boldsymbol{F}}^{-\mathrm{t}}-\bar{J}^{-1}[1-\ln \bar{J}] \overline{\mathbb{D}}\right]$

$\partial_{\bar{\Theta}} \overline{\boldsymbol{P}}_{0}=\partial_{\overline{\boldsymbol{F}}} \bar{\Psi}^{0}=-2 \bar{\alpha} \bar{\kappa}^{-1}[1-\ln \bar{J}] \overline{\boldsymbol{F}}^{-\mathrm{t}}$

$\partial_{\bar{\Theta}} \bar{\Psi}^{0}=-\bar{c}_{\bar{F}}^{0} \bar{\Theta}^{-1}$

$\partial_{\bar{\Theta}}\left(\bar{\Theta} \partial_{\bar{\Theta}} \overline{\boldsymbol{P}}_{0}: \mathrm{D}_{t} \overline{\boldsymbol{F}}\right)=-2 \bar{\alpha} \bar{\kappa} \bar{J}^{-1}[1-\ln \bar{J}] \overline{\operatorname{div}} \overline{\boldsymbol{V}}$

$\partial_{\overline{\boldsymbol{F}}}\left(\bar{\Theta} \partial_{\bar{\Theta}} \overline{\boldsymbol{P}}_{0}: \mathrm{D}_{t} \overline{\boldsymbol{F}}\right)=-2 \bar{\alpha} \bar{\kappa} \bar{\Theta}\left[\bar{J}^{-1}[\ln \bar{J}-2] \overline{\operatorname{div}} \overline{\boldsymbol{V}} \overline{\boldsymbol{F}}^{-\mathrm{t}}+\bar{J}^{-1}[1-\ln \bar{J}]\left[\mathrm{D}_{t} \overline{\boldsymbol{F}}: \overline{\mathbb{D}}+[1 / \Delta t] \overline{\boldsymbol{F}}^{-\mathrm{t}}: \overline{\mathbb{I}}\right]\right]$

$\partial_{\overline{\boldsymbol{F}}} \overline{\boldsymbol{Q}}_{0}=-\bar{J} \bar{k}_{0}\left[\overline{\boldsymbol{F}}^{-1} \cdot \overline{\boldsymbol{F}}^{-\mathrm{t}} \otimes \overline{\boldsymbol{F}}^{-\mathrm{t}}+\overline{\mathbb{B}}\right] \cdot \overline{\mathrm{Grad}} \overline{\boldsymbol{\Theta}}$

$\partial_{\overline{\mathrm{Grad}}} \bar{\Theta} \overline{\boldsymbol{Q}}_{0}=-\bar{J} \bar{k}_{0} \overline{\boldsymbol{G}}$

$\overline{\mathbb{D}}=\partial_{\overline{\boldsymbol{F}}} \overline{\boldsymbol{F}}^{-\mathrm{t}}=-\overline{\boldsymbol{F}}^{-\mathrm{t}} \otimes \overline{\boldsymbol{F}}^{-1}+[\boldsymbol{i}-\overline{\boldsymbol{i}}] \bar{\otimes} \overline{\boldsymbol{F}}^{-1} \cdot \overline{\boldsymbol{F}}^{-\mathrm{t}}$

,$\quad \overline{\mathbb{I}}=\partial_{\bar{F}} \overline{\boldsymbol{F}}=i \bar{\otimes} \overline{\boldsymbol{I}}$

$\overline{\mathbb{B}}=\partial_{\overline{\boldsymbol{F}}}\left(\overline{\boldsymbol{F}}^{-1} \cdot \overline{\boldsymbol{F}}^{-\mathrm{t}}\right)={ }^{\mathrm{t}}\left[\overline{\boldsymbol{F}}^{-1} \cdot \overline{\mathbb{D}}\right]+\overline{\boldsymbol{F}}^{-1} \cdot \overline{\mathbb{D}}$

,$\quad \overline{\boldsymbol{G}}=\overline{\boldsymbol{F}}^{-1} \cdot \overline{\boldsymbol{F}}^{-\mathrm{t}}$ 


\section{References}

[1] Altenbach, H., Eremeyev, V. A., and Morozov, N. F. (2012). Surface viscoelasticity and effective properties of thin-walled structures at the nanoscale. International Journal of Engineering Science, 59:83 - 89.

[2] Bažant, Z. P. and Jirásek, M. (2002). Nonlocal Integral Formulations of Plasticity and Damage: Survey of Progress. Journal of Engineering Mechanics, 128(11):1119-1149.

[3] Bažant, Z. P. and Xi, Y. (1991). Statistical Size Effect in QuasiBrittle Structures: II. Nonlocal Theory. Journal of Engineering Mechanics, 117(11):2623 - 2640.

[4] Benveniste, Y. (2013). Models of thin interphases and the effective medium approximation in composite media with curvilinearly anisotropic coated inclusions. International Journal of Engineering Science, 72:140 - 154.

[5] Benveniste, Y. and Miloh, T. (2001). Imperfect soft and stiff interfaces in two-dimensional elasticity. Mech. Mater., 33(6):309 - 323.

[6] Berber, S., Kwon, Y.-K., and 'anek, D. T. (2000). Unusually High Thermal Conductivity of Carbon Nanotubes. Phys. Rev. Lett., 84:4613 4616.

[7] Cahill, D. G., Ford, W. K., Goodson, K. E., Mahan, G. D., Majumdar, A., Maris, H. J., Merlin, R., and Phillpot, S. R. (2003). Nanoscale thermal transport. J. Appl. Phys., 93(2):793-818.

[8] Cammarata, R. C. (1994). Surface and interface stress effects in thin films. Prog. Surf. Sci., 46(1):1 - 38

[9] Chaboche, J.-L. (1981). Continuous damage mechanics - A tool to describe phenomena before crack initiation. Nucl. Eng. Des., 64(2):233 247.

[10] Che, J., Çagin, T., and III, W. A. G. (2000). Thermal conductivity of carbon nanotubes. Nanotechnology, 11(2):65.

[11] Daher, N. and Maugin, G. A. (1986). The method of virtual power in continuum mechanics application to media presenting singular surfaces and interfaces. Acta Mechanica, 60(3 - 4):217 - 240.

[12] Davydov, D., Javili, A., and Steinmann, P. (2013). On molecular statics and surface-enhanced continuum modeling of nano-structures. Computational Materials Science, 69(0):510 - 519

[13] de Souza Neto, E. A. and Perić, D. (1996). A computational framework for a class of fully coupled models for elastoplastic damage at finite strains with reference to the linearization aspects. Computer Methods in Applied Mechanics and Engineering, $130(1$ - 2):179 - 193.

[14] de Souza Neto, E. A., Perić, D., and Owen, D. R. J. (1998). Continuum modelling and numerical simulation of material damage at finite strains. Archives of Computational Methods in Engineering, 5(4):311 - 384.

[15] dell'Isola, F. and Romano, A. (1987). On the derivation of thermomechanical balance equations for continuous systems with a nonmaterial interface. International Journal of Engineering Science, 25:1459 - 1468.

[16] Dingreville, R., Qu, J., and Cherkaoui, M. (2005). Surface free energy and its effect on the elastic behavior of nano-sized particles, wires and films. Journal of the Mechanics and Physics of Solids, 53(8):1827 - 1854.

[17] Duan, H. L., Wang, J., Huang, Z. P., and Karihaloo, B. L. (2005a). Eshelby formalism for nano-inhomogeneities. Proceedings of the Royal Society A: Mathematical, Physical and Engineering Science, 461(2062):3335 - 3353.

[18] Duan, H. L., Wang, J., Huang, Z. P., and Karihaloo, B. L. (2005b). Size-dependent effective elastic constants of solids containing nanoinhomogeneities with interface stress. Journal of the Mechanics and Physics of Solids, 53(7):1574 - 1596.

[19] Duan, H. L., Wang, J., and Karihaloo, B. L. (2009). Theory of Elasticity at the Nanoscale. Advances in Applied Mechanics, $42: 1$ - 68.

[20] Esmaeili, A., Javili, A., and Steinmann, P. (2016a). A thermo-mechanical cohesive zone model accounting for mechanically energetic Kapitza interfaces. International Journal of Solids and Structures, 92 - 93:29 - 44.

[21] Esmaeili, A., Javili, A., and Steinmann, P. (2016b). Highly-conductive energetic coherent interfaces subject to in-plane degradation. Mathematics and Mechanics of Solids, doi: 10.1177/1081286516642818

[22] Fischer, F. D. and Svoboda, J. (2010). Stresses in hollow nanoparticles. International Journal of Solids and Structures, 47(20):2799 - 2805.

[23] Fried, E. and Gurtin, M. (2007). Thermomechanics of the interface between a body and its environment. Continuum Mech. Thermodyn., 19(5):253-271.

[24] Fried, E. and Todres, R. (2005). Mind the Gap: The Shape of the Free Surface of a Rubber-Like Material in Proximity to a Rigid Contactor. Journal of Elasticity, 80(1 - 3):97 - 151.

[25] Gurtin, M. E. and Murdoch, A. I. (1975). A continuum theory of elastic material surfaces. Archive for Rational Mechanics and Analysis, 57(4):291-323.

[26] Gurtin, M. E., Weissmüller, J., and Larché, F. (1998). A general theory of curved deformable interfaces in solids at equilibrium. Philosophical Magazine A: Physics of Condensed Matter, Structure, Defects and Mechanical Properties, 78(5):1093 - 1109.

[27] Huang, Z. P. and Sun, L. (2007). Size-dependent effective properties of a heterogeneous material with interface energy effect: From finite deformation theory to infinitesimal strain analysis. Acta Mechanica, 190(1 - 4):151 - 163.

[28] Javili, A., Kaessmair, S., and Steinmann, P. (2014). General imperfect interfaces. Computer Methods in Applied Mechanics and Engineering, $275: 76-97$

[29] Javili, A., McBride, A., and Steinmann, P. (2012). Numerical modelling of thermomechanical solids with mechanically energetic (generalised) Kapitza interfaces. Computational Materials Science, 65(0):542 - 551.

[30] Javili, A., McBride, A., and Steinmann, P. (2013). Numerical modelling of thermomechanical solids with highly conductive energetic interfaces. International Journal for Numerical Methods in Engineering, 93(5):551 - 574.

[31] Javili, A. and Steinmann, P. (2010). On thermomechanical solids with boundary structures. International Journal of Solids and Structures, 47(24):3245 - 3253 .

[32] Kachanov, L. M. (1958). Time of the rupture process under creep conditions. Izv. Akad. Nauk. S.S.R. Otd. Tech. Nauk., 8:26 - 31.

[33] Kaessmair, S., Javili, A., and Steinmann, P. (2014). Thermomechanics of solids with general imperfect coherent interfaces. Archive of Applied Mechanics, 84(9 - 11):1409 - 1426.

[34] Levitas, V. I. and Javanbakht, M. (2010). Surface tension and energy in multivariant martensitic transformations: Phase-field theory, simulations, and model of coherent interface. Phys. Rev. Lett., 105(16):165701.

[35] Moeckel, G. P. (1975). Thermodynamics of an interface. Archive for Rational Mechanics and Analysis, 57(3):255 - 280 
[36] Murdoch, A. I. (1976). A thermodynamical theory of elastic material interfaces. The Quarterly Journal of Mechanics and Applied Mathematics, 29(3):245 - 275 .

[37] Prasher, R. (2005). Predicting the Thermal Resistance of Nanosized Constrictions. Nano Lett., 5(11):2155 - 2159. Pmid: 16277444.

[38] Prasher, R. (2006). Thermal Interface Materials: Historical Perspective, Status, and Future Directions. Proceedings of the IEEE, 94(8):1571 $-1586$.

[39] Rabotnov, Y. N. (1963). On the Equation of State of Creep. Proceedings of the Institution of Mechanical Engineers, Conference Proceedings, 178(1):2-117-2-122.

[40] Sharma, P., Ganti, S., and Bhate, N. (2003). Effect of surfaces on the size-dependent elastic state of nano-inhomogeneities. Appl. Phys. Lett., 82(4):535 - 537.

[41] Simo, J. C. and Hughes, T. J. R. (1998). Computational Inelasticity. Springer-Verlag, New York.

[42] Steigmann, D. J. and Ogden, R. W. (1999). Elastic Surface-Substrate Interactions. Proceedings of the Royal Society of London A: Mathematical, Physical and Engineering Sciences, 455(1982):437 - 474.

[43] Steinmann, P. (1999). Formulation and computation of geometrically non-linear gradient damage. International Journal for Numerical Methods in Engineering, 46(5):757 - 779.

[44] Steinmann, P. (2008). On boundary potential energies in deformational and configurational mechanics. Journal of the Mechanics and Physics of Solids, 56(3):772 - 800.

[45] Steinmann, P., Miehe, C., and Stein, E. (1994). Comparison of different finite deformation inelastic damage models within multiplicative elastoplasticity for ductile materials. Computational Mechanics, 13(6):458 - 474

[46] Yvonnet, J., Mitrushchenkov, A., Chambaud, G., and He, Q.-C. (2011). Finite element model of ionic nanowires with size-dependent mechanical properties determined by ab initio calculations. Computer Methods in Applied Mechanics and Engineering, $200(5$ - 8):614 - 625. 